\title{
The Incompressible Limit of Solutions of the Two-Dimensional Compressible Euler System with Degenerating Initial Data
}

\author{
Alexandre Dutrifoy \\ Laboratoire Jacques-Louis Lions \\ Université Pierre et Marie Curie \\ Boîte courrier 187 \\ 75252 Paris Cedex 05 \\ France
}

\author{
Taoufik Hmidi \\ Centre de Mathématiques \\ École polytechnique \\ 91128 Palaiseau Cedex \\ France
}

October 4, 2005

\begin{abstract}
Using Strichartz estimates, it is possible to pass to the limit in the weakly compressible 2-D Euler system, when the Mach number $\epsilon$ tends to zero, even if the initial data are not uniformly smooth. More precisely, their norms in Sobolev spaces embedded in $C^{1}$ can be allowed to grow as small powers of $\epsilon^{-1}$.

This leads to results of convergence to solutions of the incompressible Euler system whose regularity is critical, such as vortex patches or Yudovich solutions.
\end{abstract}

\section{Introduction}

We consider a compressible fluid without viscosity, extended in the whole space $\mathbb{R}^{2}$. The Mach number, here a small positive parameter, is noted $\epsilon$; eventually, we let $\epsilon$ tend to 0 . The state of the fluid is described by the velocity field $v_{\epsilon}$ and the density $\rho_{\epsilon}$, which satisfy

$$
\left\{\begin{array}{l}
\rho_{\epsilon}\left(\partial_{t} v_{\epsilon}+v_{\epsilon} \cdot \nabla v_{\epsilon}\right)+\frac{1}{\epsilon^{2}} \nabla p_{\epsilon}=0 \\
\partial_{t} \rho_{\epsilon}+v_{\epsilon} \cdot \nabla \rho_{\epsilon}+\rho_{\epsilon} \operatorname{div} v_{\epsilon}=0 \\
\left.\left(v_{\epsilon}, \rho_{\epsilon}\right)\right|_{t=0}=\left(v_{0, \epsilon}, \rho_{0, \epsilon}\right) .
\end{array}\right.
$$

We assume the fluid to be isentropic, so the pressure is related to the density by $p_{\epsilon}=\rho_{\epsilon}^{\gamma}$, where $\gamma>1$ is a fixed parameter. If the speed of sound, defined by $c_{\epsilon}=\rho_{\epsilon} \bar{\gamma} \sqrt{\gamma} / \bar{\gamma}$ with $\bar{\gamma}=(\gamma-1) / 2$, is used as unknown instead of 
the density, then the system (1.1) becomes

$$
\left\{\begin{array}{l}
\partial_{t} v_{\epsilon}+v_{\epsilon} \cdot \nabla v_{\epsilon}+\frac{\bar{\gamma}}{\epsilon^{2}} c_{\epsilon} \nabla c_{\epsilon}=0 \\
\partial_{t} c_{\epsilon}+v_{\epsilon} \cdot \nabla c_{\epsilon}+\bar{\gamma} c_{\epsilon} \operatorname{div} v_{\epsilon}=0 \\
\left.\left(v_{\epsilon}, c_{\epsilon}\right)\right|_{t=0}=\left(v_{0, \epsilon}, c_{0, \epsilon}\right)
\end{array}\right.
$$

with $c_{0, \epsilon}=\rho_{0, \epsilon}^{\bar{\gamma}} \sqrt{\gamma} / \bar{\gamma}$.

We also assume the fluid to be weakly compressible, that is, we look for $c_{\epsilon}$ around some $c_{0}>0$. To get the simplest system possible, we set

$$
v_{\epsilon}(t, x)=\bar{\gamma} c_{0} \tilde{v}_{\epsilon}\left(\bar{\gamma} c_{0} t, x\right) \text { and } c_{\epsilon}(t, x)=c_{0}+\epsilon \bar{\gamma} c_{0} \tilde{c}_{\epsilon}\left(\bar{\gamma} c_{0} t, x\right) \text {. }
$$

Substituting these expressions in the equations (1.2) leads to

$$
\left\{\begin{array}{l}
\partial_{t} \tilde{v}_{\epsilon}+\tilde{v}_{\epsilon} \cdot \nabla \tilde{v}_{\epsilon}+\bar{\gamma} \tilde{c}_{\epsilon} \nabla \tilde{c}_{\epsilon}+\frac{1}{\epsilon} \nabla \tilde{c}_{\epsilon}=0 \\
\partial_{t} \tilde{c}_{\epsilon}+\tilde{v}_{\epsilon} \cdot \nabla \tilde{c}_{\epsilon}+\bar{\gamma} \tilde{c}_{\epsilon} \operatorname{div} \tilde{v}_{\epsilon}+\frac{1}{\epsilon} \operatorname{div} \tilde{v}_{\epsilon}=0 \\
\left.\left(\tilde{v}_{\epsilon}, \tilde{c}_{\epsilon}\right)\right|_{t=0}=\left(\tilde{v}_{0, \epsilon}, \tilde{c}_{0, \epsilon}\right),
\end{array}\right.
$$

with $\tilde{v}_{0, \epsilon}=v_{0, \epsilon} /\left(\bar{\gamma} c_{0}\right)$ and $\tilde{c}_{0, \epsilon}=\left(c_{0, \epsilon}-c_{0}\right) /\left(\epsilon \bar{\gamma} c_{0}\right)$.

We are interested in the convergence of solutions $\left(\tilde{v}_{\epsilon}, \tilde{c}_{\epsilon}\right)$ of (1.3) to global solutions $(v, c)$ of the two-dimensional incompressible Euler system

$$
\left\{\begin{array}{l}
\partial_{t} v+v \cdot \nabla v=-\nabla p \\
\operatorname{div} v=0 \\
c=0 \\
\left.v\right|_{t=0}=v_{0} .
\end{array}\right.
$$

This problem, of course, has already been studied in numerous articles; our reference list, although far from exhaustive, contains more items than we will refer to in the text $[1,6,7,8,19,20,21,22,24,26]$. Fluids evolving in different domains have been considered ( $\mathbb{R}^{N}$, torus, bounded domain), essentially under two kinds of hypotheses: in the well-prepared case [19, 20], one assumes that $\operatorname{div} \tilde{v}_{0, \epsilon} \rightarrow 0$ and $\tilde{c}_{0, \epsilon} \rightarrow 0$ as $\epsilon \rightarrow 0$; in the ill-prepared case, one only assumes that $\tilde{v}_{0, \epsilon}$ and $\tilde{c}_{0, \epsilon}$ are bounded in certain Sobolev spaces (for example) and that the incompressible part of $\tilde{v}_{0, \epsilon}$ tends to some field $v_{0}$.

In this paper we allow the initial data to be so ill-prepared that corresponding solutions can tend to a vortex patch or even a Yudovich solution. Since these solutions do not belong to the Sobolev space $H^{2+s}$ for any $s>0$, we will have to allow initial data that are not uniformly bounded in these spaces. As we will see, the dispersive effects are strong enough to deal with such particularly ill-prepared initial data. Strichartz estimates, which reflect the phenomenon of dispersion of acoustic waves, are indeed efficient tools to deal with ill-prepared initial data when the fluid is extended in the whole space; this was shown previously in the presence of a term of viscosity $[6,7]$ 
as well as in its absence $[1,26]$. Different phenomena occur in the torus or in a domain with boundary (resonances, formation of a boundary layer).

Similar results to those presented here have also been obtained about rapidly rotating fluids [9] and about solutions of the Boussinesq system and their quasigeostrophic limit [12]. These two examples are more complicated, because of the necessity of proving new dispersive estimates -instead of simply using the well-known estimates on solutions of the wave equation [15], as we do below.

Our results are fully expressed by Theorem 3 (p. 11) and Theorem 4 (p. 16), but these are perhaps a bit technical. So we thought that particular cases were worth to be formulated in the introduction.

But before, recall that even a divergence-free velocity field whose curl is, say, bounded and compactly supported does not, in general, belong to $L^{2}$ [3]. So we will have consider $\tilde{v}_{\epsilon}$ in some space $\sigma^{\prime}+L^{2}\left(\mathbb{R}^{2}\right)$, where

$$
\sigma^{\prime}\left(x_{1}, x_{2}\right)=\frac{1}{|x|^{2}}\left(\begin{array}{c}
-x_{2} \\
x_{1}
\end{array}\right) \int_{0}^{|x|} \tau g(\tau) d \tau,
$$

with $g \in C_{0}^{\infty}\left(\mathbb{R}_{0}^{+}\right)$. Such $\sigma^{\prime}$ are smooth stationary solutions of the Euler system:

$$
\partial_{t} \sigma^{\prime}=P\left(\sigma^{\prime} \cdot \nabla \sigma^{\prime}\right)=0
$$

where $P$ denotes Leray's projector onto divergence-free vector fields; they behave like $1 /|x|$ at infinity, and $\nabla \sigma^{\prime}$ belongs to $H^{r}\left(\mathbb{R}^{2}\right)$ for all $r \in \mathbb{R}$.

Convergence to Yudovich solutions is illustrated by the following theorem.

Theorem 1. Let $v_{0} \in \sigma^{\prime}+L^{2}$ be a divergence-free vector field such that

$$
\operatorname{rot} v_{0} \in L^{1} \cap L^{\infty} \text {. }
$$

Suppose that, for some $s \in] 0,1[\alpha<1$ and $\beta<1 / 12$, and some constant $C_{0}$ independent from $\epsilon$,

$$
\begin{aligned}
\left\|\operatorname{rot} \tilde{v}_{0, \epsilon}\right\|_{L^{1} \cap L^{\infty}} & \leq C_{0} \\
\left\|\operatorname{rot} \tilde{v}_{0, \epsilon}\right\|_{C^{s}} & \leq C_{0} \exp \left(\left(\ln \epsilon^{-1}\right)^{\alpha}\right), \\
\left\|\left(\tilde{v}_{0, \epsilon}-\sigma^{\prime}, \tilde{c}_{0, \epsilon}\right)\right\|_{H^{s+\frac{11}{4}}} & \leq C_{0} \epsilon^{-\beta}
\end{aligned}
$$

and that

$$
P \tilde{v}_{0, \epsilon}-v_{0} \rightarrow 0 \quad \text { in } L^{2}\left(\mathbb{R}^{2}\right) .
$$

Then the lifespans $\tilde{T}_{\epsilon}$ of the solutions of (1.3) tend to $+\infty$ as $\epsilon$ goes to zero:

$$
\tilde{T}_{\epsilon} \gtrsim \ln \ln \epsilon^{-1}
$$

the incompressible parts of the solutions tend to the Yudovich solution of the system (1.4):

$$
P \tilde{v}_{\epsilon}-v \rightarrow 0 \quad \text { in } L_{\mathrm{loc}}^{\infty}\left(\mathbb{R}^{+} ; L^{2}\right)
$$


finally, the compressible and acoustic parts of the solutions tend to zero:

$$
\left(\tilde{v}_{\epsilon}-P \tilde{v}_{\epsilon}, \tilde{c}_{\epsilon}\right) \rightarrow 0 \quad \text { in } L_{\text {loc }}^{1}\left(\mathbb{R}^{+} ; \text {Lip }\right) .
$$

An example of data $\tilde{v}_{0, \epsilon}$ to which Theorem 1 can be applied is that of regularizations of $v_{0}$. Denoting by $\rho_{k}$ the usual mollifiers, for all $k \in \mathbb{N}$, we may take

$$
P \tilde{v}_{0, \epsilon}=\rho_{k} * v_{0}
$$

if $k=k(\epsilon)$ is chosen such that

$$
k^{s} \leq C_{0} \exp \left(\left(\ln \epsilon^{-1}\right)^{\alpha}\right)
$$

and

$$
k^{s+\frac{11}{4}} \leq C_{0} \epsilon^{-\beta},
$$

condition (1.11) being evidently much stronger than condition (1.12) for small $\epsilon$. So the regularization process must be very slow. (The compressible and acoustic parts of the data don't matter as long as (1.7) is satisfied.)

In the case of regularizations of a vortex patch, we can do better than just apply Theorem 1: condition (1.11) can be dropped .

Theorem 2. Let $s \in] 0,1\left[\right.$. Let $v_{0} \in \sigma^{\prime}+L^{2}\left(\mathbb{R}^{2}\right)$ be a divergence-free vector field such that

$$
\operatorname{rot} v_{0}=\omega_{0, i} \mathbf{1}_{D}+\omega_{0, e} \mathbf{1}_{\mathbb{R}^{2} \backslash D},
$$

where $\omega_{0, i} \in C^{s}(\bar{D}), \omega_{0, e} \in C^{s} \cap L^{1}\left(\mathbb{R}^{2} \backslash D\right)$ and $D$ is a bounded domain of class $C^{1+s}$.

Suppose that

$$
\tilde{v}_{0, \epsilon}=\rho_{\left\lfloor\epsilon^{\left.-\frac{1}{15}\right\rfloor}\right.} * v_{0}+w_{0, \epsilon},
$$

where $\operatorname{rot} w_{0, \epsilon}=0$ for each $\epsilon$, and that

$$
\left\|w_{0, \epsilon}\right\|_{H^{s+\frac{11}{4}}}+\left\|\tilde{c}_{0, \epsilon}\right\|_{H^{s+\frac{11}{4}}} \lesssim \epsilon^{-\frac{1}{9}} .
$$

Then the lifespans of the solutions of (1.3) grow as $\ln \ln \epsilon^{-1}$; their incompressible parts tend, in $L_{\mathrm{loc}}^{\infty}\left(\mathbb{R}^{+} ; \sigma^{\prime}+L^{2}\right)$, to the vortex patch solution of the incompressible Euler system with datum $v_{0}$, and their compressible and acoustic parts tend to zero in $L_{\mathrm{loc}}^{1}\left(\mathbb{R}^{+} ; \mathrm{Lip}\right)$.

The explanation of this improvement is that actually, the $C^{s}$-norm in assumption (1.6) may be replaced by a weaker norm - exploiting a kind of $C^{s}$ regularity in only one direction related to the structure of a vortex patch (see Section 4).

\section{Plan of the article}

In the next section, we reformulate the system one last time, mainly for the convenience of notations, but also to stress its similarity with the other systems for which analogous results have been obtained $[12,9]$. 
After that, we recall some basic a priori estimates and prove Theorem 1 (Section 3).

In Section 4, we show how to control the lifespans under slightly weaker assumptions and how this more elaborate line of reasoning (Theorems 3 and 4) actually yields the theorems stated in the introduction.

The statements of Theorems 3 and 4 involve Besov spaces, accordingly with what was announced in our note [13]. However, the reader unwilling to dwell on Besov spaces should not be deterred from reading Section 4 , for the loss of precision in the last two theorems is not so great if $B_{2, q}^{s+\frac{11}{4}}$ and $B_{2,1}^{\frac{11}{4}}$ are, for example, both replaced by $H^{s+\frac{11}{4}}$ (in particular, they still imply Theorem 2).

Finally, we give in appendix a fairly self-contained proof of all required estimates.

\section{Reformulation of the system}

We set

$$
\sigma=\left(\begin{array}{c}
\sigma^{1} \\
\sigma^{\prime 2} \\
0
\end{array}\right), \quad U_{\epsilon}=\left(\begin{array}{c}
\tilde{v}_{\epsilon}^{1} \\
\tilde{v}_{\epsilon}^{2} \\
\tilde{c}_{\epsilon}
\end{array}\right)-\sigma, \quad U_{\epsilon}^{\prime}=\left(\begin{array}{c}
U_{\epsilon}^{1} \\
U_{\epsilon}^{2}
\end{array}\right) ;
$$

then (1.3) may be rewritten

$$
\left\{\begin{array}{l}
\partial_{t} U_{\epsilon}+\left(U_{\epsilon}^{\prime}+\sigma^{\prime}\right) \cdot \nabla\left(U_{\epsilon}+\sigma\right)+\left(\bar{\gamma} U_{\epsilon}^{3}+\frac{1}{\epsilon}\right) B(D) U_{\epsilon}=0 \\
\left.U_{\epsilon}\right|_{t=0}=U_{0, \epsilon}
\end{array}\right.
$$

where

$$
B(D)=\left(\begin{array}{ccc}
0 & 0 & \partial_{1} \\
0 & 0 & \partial_{2} \\
\partial_{1} & \partial_{2} & 0
\end{array}\right)
$$

and

$$
U_{0, \epsilon}=\left(\begin{array}{c}
\tilde{v}_{0, \epsilon}^{1} \\
\tilde{v}_{0, \epsilon}^{2} \\
\tilde{c}_{0, \epsilon}
\end{array}\right)-\sigma .
$$

Solving (1.3) in $\sigma+H^{s}$ is equivalent to solving (2.1) in $H^{s}$. Since we always assume $s>2$, doing this locally in time is not a problem, essentially because (2.1) is symmetric and all derivatives of $\sigma$ belong to $L^{2}$.

Orthonormal eigenvectors of $B(\xi)$ are

$$
V_{0}(\xi)=\frac{1}{|\xi|}\left(\begin{array}{c}
-\xi^{2} \\
\xi^{1} \\
0
\end{array}\right)
$$


corresponding to the eigenvalue 0 , and

$$
V_{ \pm 1}(\xi)=\frac{\sqrt{2}}{2|\xi|}\left(\begin{array}{c} 
\pm \xi^{1} \\
\pm \xi^{2} \\
|\xi|
\end{array}\right)
$$

corresponding to the eigenvalues $\pm i|\xi|$. We shall use the projectors

$$
P_{0}=V_{0}(D){ }^{t} V_{0}(D) \text { and } P_{ \pm 1}=V_{ \pm 1}(D)^{t} V_{ \pm 1}(D) .
$$

One can check that

$$
P_{0}\left(\begin{array}{c}
v^{1} \\
v^{2} \\
c
\end{array}\right)=\left(\begin{array}{c}
\text { incompressible part of } v \\
0
\end{array}\right),
$$

while

$$
Q\left(\begin{array}{c}
v^{1} \\
v^{2} \\
c
\end{array}\right) \stackrel{\text { def }}{=}\left(P_{1}+P_{-1}\right)\left(\begin{array}{c}
v^{1} \\
v^{2} \\
c
\end{array}\right)=\left(\begin{array}{c}
\text { compressible part of } v \\
c
\end{array}\right) .
$$

\section{Proof of Theorem 1}

In addition to the well-known logarithmic inequality between the Lipschitzian norm of a divergence-free vector fields and some Hölderian norm of its curl-here

$$
\begin{aligned}
\left\|P_{0} U_{\epsilon}(t)\right\|_{\text {Lip }} & =\left\|P_{0} U_{\epsilon}(t)\right\|_{L^{\infty}}+\left\|\nabla P_{0} U_{\epsilon}(t)\right\|_{L^{\infty}} \\
& \lesssim\left\|\Omega_{\epsilon}(t)\right\|_{L^{1} \cap L^{\infty}}+\left\|\Omega_{\epsilon}(t)\right\|_{L^{\infty}} \ln \left(e+\frac{\left\|\Omega_{\epsilon}(t)\right\|_{C^{s}}}{\left\|\Omega_{\epsilon}(t)\right\|_{L^{\infty}}}\right)
\end{aligned}
$$

for any $s \in] 0,1[$ - we will need three kinds of very classical estimates, namely

- an energy estimate [23]:

$$
\begin{aligned}
\left\|U_{\epsilon}(t)\right\|_{H^{s+\frac{11}{4}}} \lesssim\left\|U_{0, \epsilon}\right\|_{H^{s+\frac{11}{4}}}+C_{\sigma} t+C_{\sigma} \int_{0}^{t}\left\|U_{\epsilon}\left(t^{\prime}\right)\right\|_{\text {Lip }} d t^{\prime} \\
+\int_{0}^{t}\left(C_{\sigma}+\left\|U_{\epsilon}\left(t^{\prime}\right)\right\|_{\text {Lip }}\right)\left\|U_{\epsilon}\left(t^{\prime}\right)\right\|_{H^{s+\frac{11}{4}}} d t^{\prime}
\end{aligned}
$$

if

$$
C_{\sigma} \geq\|\sigma\|_{\text {Lip }}+\left\|\sigma^{\prime} \cdot \nabla \sigma\right\|_{H^{r}}+\|\nabla \sigma\|_{H^{r}}
$$


- dispersive estimates [15]:

$$
\begin{aligned}
& \left\|\nabla P_{ \pm 1} U_{\epsilon}\right\|_{L_{t}^{4+\frac{8}{p-2}}\left(L^{p}\right)} \lesssim \epsilon^{\frac{1}{4}-\frac{1}{2 p}}\left(\left\|P_{ \pm 1} U_{0, \epsilon}\right\|_{H^{\frac{7}{4}}}\right. \\
& \left.\quad+\int_{0}^{t}\left(C_{\sigma}+C_{\sigma}\left\|U_{\epsilon}\left(t^{\prime}\right)\right\|_{H^{\frac{11}{4}}}+\left\|U_{\epsilon}\left(t^{\prime}\right)\right\|_{\text {Lip }}\left\|U_{\epsilon}\left(t^{\prime}\right)\right\|_{H^{\frac{11}{4}}}\right) d t^{\prime}\right)
\end{aligned}
$$

for $2<p<+\infty$, and

$$
\begin{aligned}
& \left\|P_{ \pm 1} U_{\epsilon}\right\|_{L_{t}^{4}\left(C^{s+1}\right)} \lesssim \epsilon^{\frac{1}{4}}\left(\left\|P_{ \pm 1} U_{0, \epsilon}\right\|_{H^{s+\frac{7}{4}}}\right. \\
& \left.+\int_{0}^{t}\left(C_{\sigma}+C_{\sigma}\left\|U_{\epsilon}\left(t^{\prime}\right)\right\|_{H^{s+\frac{11}{4}}}+\left\|U_{\epsilon}\left(t^{\prime}\right)\right\|_{\text {Lip }}\left\|U_{\epsilon}\left(t^{\prime}\right)\right\|_{H^{s+\frac{11}{4}}}\right) d t^{\prime}\right)
\end{aligned}
$$

if

$$
C_{\sigma} \geq\left\|\sigma^{\prime} \cdot \nabla \sigma\right\|_{H^{s+\frac{11}{4}}}+\|\nabla \sigma\|_{H^{s+\frac{11}{4}}}+\|\sigma\|_{C^{s+\frac{11}{4}}}
$$

- estimates on the curls of $\tilde{v}_{\epsilon}$ (see Appendix C):

$$
\left\|\Omega_{\epsilon}(t)\right\|_{L^{p}} \leq\left\|\Omega_{0, \epsilon}\right\|_{L^{p}} e^{\left(1+\frac{1}{p}\right) \int_{0}^{t}\left\|\operatorname{div} U_{\epsilon}^{\prime}\left(t^{\prime}\right)\right\|_{L} \infty d t^{\prime}},
$$

for all $p \in[1,+\infty]$, and

$$
\begin{aligned}
\left\|\Omega_{\epsilon}(t)\right\|_{C^{s}} \lesssim\left\|\Omega_{0, \epsilon}\right\|_{C^{s}} & +\int_{0}^{t}\left\|\Omega_{\epsilon}\left(t^{\prime}\right)\right\|_{L^{\infty}}\left\|\operatorname{div} U_{\epsilon}^{\prime}\left(t^{\prime}\right)\right\|_{C^{s}} d t^{\prime} \\
& +\int_{0}^{t}\left(\|\sigma\|_{\text {Lip }}+\left\|U_{\epsilon}^{\prime}\left(t^{\prime}\right)\right\|_{\text {Lip }}\right)\left\|\Omega_{\epsilon}\left(t^{\prime}\right)\right\|_{C^{s}} d t^{\prime}
\end{aligned}
$$

In view of (3.2), it is clear that

$$
V_{\epsilon}(t) \stackrel{\text { def }}{=} \int_{0}^{t}\left\|U_{\epsilon}\left(t^{\prime}\right)\right\|_{\text {Lip }} d t^{\prime}
$$

controls the lifespans of smooth solutions of (2.1).

A consequence of (3.2) and of the assumptions on the initial data is that

$$
\begin{aligned}
&\left\|U_{\epsilon}(t)\right\|_{H^{s+\frac{11}{4}}} \lesssim C_{0} \epsilon^{-\beta}+C_{\sigma} t+C_{\sigma} V_{\epsilon}(t) \\
&+\int_{0}^{t}\left(C_{\sigma}+\left\|U_{\epsilon}\left(t^{\prime}\right)\right\|_{\mathrm{Lip}}\right)\left\|U_{\epsilon}\left(t^{\prime}\right)\right\|_{H^{s+\frac{11}{4}}} d t^{\prime}
\end{aligned}
$$

and therefore

$$
\begin{aligned}
\left\|U_{\epsilon}(t)\right\|_{H^{s+\frac{11}{4}}} & \lesssim\left(C_{0} \epsilon^{-\beta}+C_{\sigma} t+C_{\sigma} V_{\epsilon}(t)\right) e^{C\left(C_{\sigma} t+V_{\epsilon}(t)\right)} \\
& \lesssim C_{0} \epsilon^{-\beta} e^{C C_{\sigma}\left(t+V_{\epsilon}(t)\right)}
\end{aligned}
$$


if we suppose, as we may, that $\epsilon \leq 1$ and $C_{0} \geq 1$. Introducing this in (3.4), we get

$$
\begin{aligned}
\int_{0}^{t}\left\|Q U_{\epsilon}\left(t^{\prime}\right)\right\|_{\text {Lip }} d t^{\prime} & \lesssim \int_{0}^{t}\left\|Q U_{\epsilon}\left(t^{\prime}\right)\right\|_{C^{s+1}} d t^{\prime} \\
& \lesssim t^{\frac{3}{4}} \epsilon^{\frac{1}{4}}\left(C_{0} \epsilon^{-\beta}+C_{\sigma} t+\left(C_{\sigma} t+V_{\epsilon}(t)\right) C_{0} \epsilon^{-\beta} e^{C C_{\sigma}\left(t+V_{\epsilon}(t)\right)}\right) \\
& \lesssim C_{0} t^{\frac{3}{4}} \epsilon^{\frac{1}{4}-\beta} e^{C C_{\sigma}\left(t+V_{\epsilon}(t)\right)} \stackrel{\text { def }}{=} g_{\epsilon}(t) .
\end{aligned}
$$

We suppose in the sequel that $g_{\epsilon}(t) \leq 1$, which is true at least for small $t$.

Now let us turn to the quantities in the right member of (3.1). The estimates (3.5) give

$$
\left\|\Omega_{\epsilon}(t)\right\|_{L^{1} \cap L^{\infty}} \leq C_{0} e^{C g_{\epsilon}(t)} \lesssim C_{0} .
$$

Then the estimate (3.6) gives

$\left\|\Omega_{\epsilon}(t)\right\|_{C^{s}} \lesssim C_{0} \exp \left(\left(\ln \epsilon^{-1}\right)^{\alpha}\right)+C_{0} g_{\epsilon}(t)+\int_{0}^{t}\left(C_{\sigma}+\left\|U_{\epsilon}\left(t^{\prime}\right)\right\|_{\text {Lip }}\right)\left\|\Omega_{\epsilon}\left(t^{\prime}\right)\right\|_{C^{s}} d t^{\prime}$,

so

$$
\left\|\Omega_{\epsilon}(t)\right\|_{C^{s}} \lesssim C_{0} e^{\left(\ln \epsilon^{-1}\right)^{\alpha}+C\left(C_{\sigma} t+V_{\epsilon}(t)\right)}
$$

by Gronwall's lemma. Thus

$$
\begin{aligned}
\left\|P_{0} U_{\epsilon}(t)\right\|_{\text {Lip }} \lesssim & C_{0}+C_{0} \ln \left(e+C_{0} e^{\left(\ln \epsilon^{-1}\right)^{\alpha}+C\left(C_{\sigma} t+V_{\epsilon}(t)\right)}\right) \\
\lesssim & C_{0} \ln \left(e+C_{0}\right)+C_{0}\left(\ln \epsilon^{-1}\right)^{\alpha}+C_{0} C_{\sigma} t \\
& +C_{0} \int_{0}^{t}\left\|P_{0} U_{\epsilon}\left(t^{\prime}\right)\right\|_{\text {Lip }} d t^{\prime} .
\end{aligned}
$$

A new application of Gronwall's lemma gives

$$
\left\|P_{0} U_{\epsilon}(t)\right\|_{\text {Lip }} \lesssim C_{0}\left(\ln \left(e+C_{0}\right)+\left(\ln \epsilon^{-1}\right)^{\alpha}+C_{\sigma} t\right) e^{C C_{0} t},
$$

hence

$$
\begin{aligned}
V_{\epsilon}(t) & \leq C C_{0}\left(\ln \left(e+C_{0}\right)+\left(\ln \epsilon^{-1}\right)^{\alpha}+C_{\sigma} t\right) e^{C C_{0} t}+g_{\epsilon}(t) \\
& \leq\left(\ln \epsilon^{-1}\right)^{\alpha} e^{C C_{0}\left(C_{\sigma}+1\right)(t+1)},
\end{aligned}
$$

if $\epsilon \leq 1 / e$ and as long as $g_{\epsilon}(t) \leq 1$.

So $g_{\epsilon}(t) \leq 1$ actually implies

$$
\begin{aligned}
g_{\epsilon}(t) & \leq C_{0} t^{\frac{3}{4}} \epsilon^{\frac{1}{4}-\beta} e^{\left(\ln \epsilon^{-1}\right)^{\alpha} e^{C C_{0}\left(C_{\sigma}+1\right)(t+1)}} \\
& \leq \epsilon^{\frac{1}{4}-\beta} e^{\left(\ln \epsilon^{-1}\right)^{\alpha} e^{C C_{0}\left(C_{\sigma}+1\right)(t+1)}},
\end{aligned}
$$

this last expression being not greater than $\epsilon^{\frac{1}{8}-\frac{\beta}{2}}$ if

$$
C C_{0}\left(C_{\sigma}+1\right)(t+1) \leq \ln \left(\frac{1}{8}-\frac{\beta}{2}\right)+(1-\alpha) \ln \ln \epsilon^{-1} ;
$$


thus (1.8) and (1.10) are proved.

The convergence part of Theorem 1 is proved in much the same way as Yudovich's theorem itself [3].

Let $T>0$ be fixed. We have just seen that $\tilde{T}_{\epsilon} \geq T$ and $g_{\epsilon}(T) \leq \epsilon^{\frac{1}{8}-\frac{\beta}{2}}$ if $\epsilon$ is sufficiently small. Let $\eta>0$ to be determined, and assume that

$$
\epsilon_{1}<\epsilon \text { and } \epsilon_{2}<\epsilon \Rightarrow\left\|P_{0} U_{0, \epsilon_{1}}-P_{0} U_{0, \epsilon_{2}}\right\|_{L^{2}} \leq \eta .
$$

Let $\left.\epsilon_{1}, \epsilon_{2} \in\right] 0, \epsilon\left[\right.$; set $U_{1}=U_{\epsilon_{1}}, U_{2}=U_{\epsilon_{2}}$ and $U=U_{1}-U_{2}$. Then

$$
\left\{\begin{array}{l}
\partial_{t} P_{0} U+P_{0}\left(\left(U_{1}^{\prime}+\sigma^{\prime}\right) \cdot \nabla U+U^{\prime} \cdot \nabla\left(U_{2}+\sigma\right)\right)=0 \\
\left.P_{0} U\right|_{t=0}=P_{0} U_{0, \epsilon_{1}}-P_{0} U_{0, \epsilon_{2}}
\end{array}\right.
$$

follows from (2.1), because $P_{0}\left(U_{\epsilon}^{3} B(D) U_{\epsilon}\right)=P_{0}\left(B(D) U_{\epsilon}\right)=0$ for all $\epsilon$. Hence,

$$
\begin{aligned}
\partial_{t}\left\|P_{0} U(t)\right\|_{L^{2}}^{2} \lesssim & f_{1}(t)\left\|P_{0} U(t)\right\|_{L^{2}}^{2}+f_{2}(t)\left\|P_{0} U(t)\right\|_{L^{2}} \\
& +\int_{\mathbb{R}^{2}}\left|P_{0} U(t, x)\right|^{2}\left|\nabla\left(P_{0} U_{2}+\sigma\right)(t, x)\right| d x
\end{aligned}
$$

with

$$
f_{1}(t)=\left\|\operatorname{div} U_{1}^{\prime}(t)\right\|_{L^{\infty}}+\left\|\nabla Q U_{2}(t)\right\|_{L^{\infty}}
$$

and

$$
\begin{aligned}
f_{2}(t)= & \left\|U_{1}^{\prime}(t)\right\|_{L^{2}}\|\nabla Q U(t)\|_{L^{\infty}}+\|Q U(t)\|_{L^{\infty}}\left\|\nabla\left(U_{2}(t)+\sigma\right)\right\|_{L^{2}} \\
& +\left\|\sigma^{\prime}\right\|_{L^{\frac{2 p}{p-2}}}\|\nabla Q U(t)\|_{L^{p}} .
\end{aligned}
$$

Since, for all $b>1$,

$$
\left\|\nabla\left(P_{0} U_{2}(t)+\sigma\right)\right\|_{L^{b}} \lesssim b\left\|\Omega_{\epsilon_{2}}(t)\right\|_{L^{b}} \lesssim C_{0} b
$$

- because $\left(P_{0} U_{2}(t)+\sigma\right)^{3}=0$, and $\left(P_{0} U_{2}(t)+\sigma\right)^{\prime}$ is divergence-free and its curl is $\Omega_{\epsilon_{2}}-$, the integral in (3.7) is bounded by

$$
C_{0} b\left\|P_{0} U(t)\right\|_{L^{\frac{2 b}{b-1}}}^{2},
$$

itself bounded by

$$
C_{0} b\left\|P_{0} U(t)\right\|_{L^{2}}^{2\left(1-\frac{1}{b}\right)}\left\|P_{0} U(t)\right\|_{L^{\infty}}^{\frac{2}{b}} .
$$

But - for the same reason-

$$
\begin{aligned}
\left\|P_{0} U(t)\right\|_{L^{\infty}}^{\frac{2}{b}} & \lesssim\left(\left\|\chi(D) P_{0} U(t)\right\|_{L^{\infty}}+\left\|\Omega_{\epsilon_{1}}(t)-\Omega_{\epsilon_{2}}(t)\right\|_{L^{\infty}}\right)^{\frac{2}{b}} \\
& \lesssim\left(\left\|P_{0} U(t)\right\|_{L^{2}}+C_{0}\right)^{\frac{2}{b}} \\
& \lesssim C_{0},
\end{aligned}
$$


so, as long as $\left\|P_{0} U(t)\right\|_{L^{2}}$ is smaller than 1 ,

$$
\partial_{t}\left\|P_{0} U(t)\right\|_{L^{2}}^{2} \lesssim f_{1}(t)+f_{2}(t)+C_{0} b\left(\left\|P_{0} U(t)\right\|_{L^{2}}^{2}\right)^{1-\frac{1}{b}},
$$

for all $b>1$. Note that $\left\|f_{1}+f_{2}\right\|_{L_{T}^{1}} \rightarrow 0$ when $\epsilon \rightarrow 0$. Indeed, in addition of (4.2), we have

$$
\|Q U\|_{L_{T}^{1}\left(L^{\infty}\right)}\left\|\nabla\left(U_{2}(t)+\sigma\right)\right\|_{L_{T}^{\infty}\left(L^{2}\right)} \lesssim \epsilon^{\frac{1}{8}-\frac{\beta}{2}} \epsilon^{-\beta}
$$

and

$$
\|\nabla Q U\|_{L_{T}^{1}\left(L^{p}\right)} \lesssim \epsilon^{\frac{1}{8}-\frac{\beta}{2}-\frac{1}{2 p}}
$$

for all $p>2$. Now we can conclude thanks to Osgood lemma [3, p. 91]: if we set $J_{\eta}=\eta+\left\|P_{0} U(t)\right\|_{L^{2}}^{2}\left(\right.$ with $\eta>0$, small) and choose $b=1-\ln J_{\eta}$ in (3.8), we get

$$
J_{\eta}^{\prime} \lesssim f_{1}+f_{2}+C_{0}\left(1-\ln J_{\eta}\right) J_{\eta}
$$

which leads, after integration and exponentiations, to

$$
J_{\eta}(t) \leq\left(J_{\eta}(0)+C\left\|f_{1}+f_{2}\right\|_{L_{T}^{1}}\right)^{e^{-C C_{0} t}} e^{1-e^{-C C_{0} t}} ;
$$

so

$$
\left\|P_{0} U\right\|_{L_{T}^{\infty}\left(L^{2}\right)} \leq(2 \eta)^{e^{-C C_{0} T}} e
$$

if $\eta$ makes the right member of (3.9) not larger than 1 and $\epsilon$ is sufficiently small.

Since $T$ may be arbitrarily large and $\eta$ arbitrarily small, $P_{0} U_{\epsilon}$ converges to some $V=P_{0} V$ in $L_{\mathrm{loc}}^{\infty}\left(\mathbb{R}^{+} ; L^{2}\left(\mathbb{R}^{2}\right)\right)$. Each $P_{0} U_{\epsilon}$ satisfies

$$
\left\{\begin{array}{l}
\partial_{t} P_{0} U_{\epsilon}+P_{0}\left(\left\langle\nabla,\left(U_{\epsilon}^{\prime}+\sigma^{\prime}\right) \otimes\left(U_{\epsilon}+\sigma\right)\right\rangle-\left(U_{\epsilon}+\sigma\right) \operatorname{div} U_{\epsilon}^{\prime}\right)=0 \\
\left.\left(P_{0} U_{\epsilon}+\sigma\right)\right|_{t=0}=P_{0} U_{0, \epsilon}+\sigma
\end{array}\right.
$$

and $Q U_{\epsilon} \rightarrow 0$ in $L_{\text {loc }}^{1}\left(\mathbb{R}^{+} ; \operatorname{Lip}\left(\mathbb{R}^{2}\right)\right)$, so $V$ is a solution of

$$
\left\{\begin{array}{l}
\partial_{t} V+P_{0}\left(\left\langle\nabla,\left(V^{\prime}+\sigma^{\prime}\right) \otimes(V+\sigma)\right\rangle\right)=0 \\
\left.V\right|_{t=0}=U_{0}+\sigma
\end{array}\right.
$$

As

$$
\begin{aligned}
\left\|\operatorname{rot}\left(V^{\prime}(t)+\sigma^{\prime}\right)\right\|_{L^{1} \cap L^{\infty}} & \leq \limsup _{\epsilon \rightarrow 0}\left\|\Omega_{\epsilon}(t)\right\|_{L^{1} \cap L^{\infty}} \\
& \lesssim C_{0},
\end{aligned}
$$

for all $t \in \mathbb{R}^{+}$, we must have $V^{\prime}+\sigma^{\prime}=v$, by virtue of the uniqueness part of Yudovich theorem. 


\section{Proofs of Theorems 3 and 4}

Although we are in dimension 2, the formalism of 3-D vortex patches [14], slightly adapted, shall be convenient. We will say that a system of continuous vector fields $W=\left\{w^{\nu} ; \nu=1, \ldots, N\right\}$ is admissible if

$$
[W]^{-1} \stackrel{\text { def }}{=}\left(\frac{1}{N} \sum_{\nu=1}^{N}\left|w^{\nu}\right|\right)^{-1}
$$

is bounded. We will also note

$$
V_{\epsilon}(t)=\int_{0}^{t}\left\|U_{\epsilon}\left(t^{\prime}\right)\right\|_{\text {Lip }} d t^{\prime}
$$

and denote by $X_{s}(W, \Omega)$, for any $\left.s \in\right] 0,1[$ and any scalar function $\Omega$, the quantity

$$
\|\Omega\|_{L^{\infty}}+\left\|[W]^{-1}\right\|_{L^{\infty}}+\sum_{\nu=1}^{N}\left\|w^{\nu}\right\|_{C^{s}}+\sum_{\nu=1}^{N}\left\|\operatorname{div}\left(w^{\nu} \Omega\right)\right\|_{C^{s-1}} .
$$

Since $\operatorname{div}\left(w^{\nu} \Omega\right)$ is some kind of substitute to $w^{\nu} \cdot \nabla \Omega$ - they are equal if $\operatorname{div} w^{\nu}=0$, and the latter is sometimes more difficult to define one may think of $X_{s}(W, \Omega)$ as a norm related to $\Omega$ 's regularity in the directions indicated by the vectors of $W$.

If $\operatorname{rot} v_{0}$ is given by (1.13), then an admissible system $W_{0}=\left\{w_{0}^{1}, w_{0}^{2}\right\}$ of two $C^{s}$ vector fields, tangent to $\partial D$, can be constructed, such that $\operatorname{div}\left(w_{0}^{\nu} \operatorname{rot} v_{0}\right) \in C^{s-1}$, for $\nu=1,2$; this is a way to express that the vortex patch has some tangential regularity. We will recall the facts that we need to borrow from the theory of vortex patches as we go along.

Now we can state the real theorem. Its main purpose is to describe the conditions under which the times of existence of the solutions of (2.1) can be bounded from below; it does not say anything about the convergence of these solutions (for that, see Theorem 4 below).

Theorem 3. Let $W_{0}=\left\{w_{0}^{\nu} ; \nu=1, \ldots, N\right\}$ be an admissible system of $C^{s}$ vector fields, with $s \in] 0,1\left[\right.$. Suppose that for some constant $C_{0}$ greater than

$$
\left\|\sigma^{\prime} \cdot \nabla \sigma\right\|_{B_{2, q}^{s+\frac{11}{4}}}+\|\nabla \sigma\|_{B_{2, q}^{s+\frac{11}{4}}}+\|\sigma\|_{C^{s+\frac{11}{4}}}+1
$$

the initial data of (2.1) are bounded as follows:

$$
\begin{gathered}
\left\|U_{0, \epsilon}\right\|_{L^{2}} \leq C_{0}, \\
\left\|\Omega_{0, \epsilon}\right\|_{L^{\infty}}+\left\|\left[W_{0}\right]^{-1}\right\|_{L^{\infty}}+\sum_{\nu=1}^{N}\left\|w_{0}^{\nu}\right\|_{C^{s}} \leq C_{0}, \\
\sum_{\nu=1}^{N}\left\|\operatorname{div}\left(w_{0}^{\nu} \Omega_{0, \epsilon}\right)\right\|_{C^{s-1}} \leq C_{\epsilon}, \\
\left\|U_{0, \epsilon}\right\|_{B_{2,1}^{\frac{11}{4}} \leq C_{0} \epsilon^{-\alpha}, \quad\left\|U_{0, \epsilon}\right\|_{B_{2, q}^{s+\frac{11}{4}}} \leq C_{0} \epsilon^{-\beta},}
\end{gathered}
$$


with $q \in[1,4]$ and

$$
0 \leq \alpha \leq \beta<\frac{1}{4}
$$

and where $C_{\epsilon}$ is a non-increasing function of $\epsilon$.

Then, for all $\mu \in] 0, \frac{1}{4}-\alpha\left[\right.$, the lifespan $T_{\epsilon}$ of the solution $U_{\epsilon}$ is bounded from below by

$$
T_{\epsilon}^{(\mu)}=\min \left(C_{0}^{-\frac{8}{3}} \epsilon^{\frac{4}{3}\left(\beta-\frac{1}{4}\right)}, \frac{1}{C C_{0}} \ln \frac{\ln \left(\epsilon^{\alpha+\mu-\frac{1}{4}}\right)}{\ln \left(e+C_{\epsilon}\right)}-1\right) .
$$

Moreover,

$$
V_{\epsilon}(t) \leq e^{C C_{0}(t+1)} \ln \left(e+C_{\epsilon}\right)
$$

for all $t \in\left[0, T_{\epsilon}^{(\mu)}\right]$, and

$$
\left\|P_{ \pm 1} U_{\epsilon}\right\|_{L_{T_{\epsilon}^{1}(\mu)}(\operatorname{Lip})} \leq \epsilon^{\mu} .
$$

This theorem is interesting if $C_{\epsilon}$ does not grow too fast as $\epsilon \rightarrow 0$, so that $T_{\epsilon}^{(\mu)} \rightarrow \infty$. In particular, it applies neatly if the data are regularizations of a vortex patch, because then $C_{\epsilon} \leq C_{0}^{\prime}$ for some constant $C_{0}^{\prime}$ independent of $\epsilon$ : this is the first example in the introduction (Theorem 2). Indeed, if $\operatorname{div}\left(w_{0}^{\nu} \operatorname{rot} v_{0}\right) \in C^{s-1}$, then

$$
\begin{aligned}
\operatorname{div}\left(w_{0}^{\nu} \operatorname{rot} \tilde{v}_{0, \epsilon}\right) & =\operatorname{div}\left(w_{0}^{\nu}\left(\operatorname{rot} \rho_{\left\lfloor\epsilon^{-\frac{1}{15}}\right\rfloor} * v_{0}+\operatorname{rot} w_{0, \epsilon}\right)\right) \\
& =\operatorname{div}\left(w_{0}^{\nu} \rho_{\left\lfloor\epsilon^{-\frac{1}{15}}\right\rfloor} * \operatorname{rot} v_{0}\right) \\
& \leq C \operatorname{div}\left(w_{0}^{\nu} * \operatorname{rot} v_{0}\right),
\end{aligned}
$$

with $C$ independent of $\epsilon$.

If the data are regularizations of a field without tangential regularity, as in the case of general Yudovich solutions, we can still gain control on $C_{\epsilon}$, but only by reducing the speed of regularization so drastically that the assumption (1.6) is satisfied. Then Theorem 3 may be applied with $W_{0}=\{(1,0),(0,1)\}$ and $C_{\epsilon}=C \exp \left(\left(\ln \epsilon^{-1}\right)^{\alpha}\right)$.

Proof of Theorem 3. Let $W_{\epsilon}(t)=\left\{w_{\epsilon}^{\nu}(t) ; \nu=1, \ldots, N\right\}$ with $w_{\epsilon}^{\nu}$ defined, for every $\nu$, as the solution of

$$
\left\{\begin{array}{l}
\partial_{t} w_{\epsilon}^{\nu}+\left(U_{\epsilon}^{\prime}+\sigma^{\prime}\right) \cdot \nabla w_{\epsilon}^{\nu}=w_{\epsilon}^{\nu} \cdot \nabla\left(U_{\epsilon}^{\prime}+\sigma^{\prime}\right) \\
\left.w_{\epsilon}^{\nu}\right|_{t=0}=w_{0}^{\nu}
\end{array}\right.
$$

In other words, $w_{\epsilon}^{\nu}(t)$ is the image of $w_{0}^{\nu}$ by the differential of the flow $\psi_{\epsilon}$ of the vector field $\tilde{v}_{\epsilon}=U_{\epsilon}^{\prime}+\sigma^{\prime}: w_{\epsilon}^{\nu}\left(t, \psi_{\epsilon}(t, \cdot)\right)=w_{0}^{\nu} \cdot \nabla \psi_{\epsilon}(t, \cdot)$. (This definition is natural in the context of vortex patches, for it ensures in the 
simplest manner that if $w_{0}^{\nu}$ is tangent to $\partial D$, then $w_{\epsilon}^{\nu}(t)$ will be tangent to $\psi_{\epsilon}(t, \partial D)$.) From (4.3), one can deduce that

$$
\left\|\left[W_{\epsilon}(t)\right]^{-1}\right\|_{L^{\infty}} \lesssim\left\|\left[W_{0}\right]^{-1}\right\|_{L^{\infty}} e^{C \int_{0}^{t}\left\|\tilde{v}_{\epsilon}\left(t^{\prime}\right)\right\|_{\text {Lip }} d t^{\prime}},
$$

which implies that $W_{\epsilon}^{\nu}(t)$ stays admissible as long as it is defined [14, p. 406]. Consequently, the estimate

$$
\left\|\nabla \tilde{v}_{\epsilon}(t)\right\|_{L^{\infty}} \lesssim\left\|\Omega_{\epsilon}(t)\right\|_{L^{\infty}} \ln \left(e+\frac{X_{\epsilon}(t)}{\left\|\Omega_{\epsilon}(t)\right\|_{L^{\infty}}}\right),
$$

with $X_{\epsilon} \stackrel{\text { def }}{=} X_{s}\left(W_{\epsilon}, \Omega_{\epsilon}\right)$, stays valid, too [14, p. 396-401]. So

$$
\begin{aligned}
\left\|P_{0} U_{\epsilon}(t)\right\|_{\text {Lip }} & \lesssim\left\|\chi(D) P_{0} U_{\epsilon}(t)\right\|_{L^{\infty}}+\left\|\nabla P_{0} U_{\epsilon}(t)\right\|_{L^{\infty}} \\
& \lesssim\left\|U_{\epsilon}(t)\right\|_{L^{2}}+\|\nabla \sigma\|_{L^{\infty}}+\left\|\Omega_{\epsilon}(t)\right\|_{L^{\infty}} \ln \left(e+\frac{X_{\epsilon}(t)}{\left\|\Omega_{\epsilon}(t)\right\|_{L^{\infty}}}\right)
\end{aligned}
$$

for all $t \in\left[0, \tilde{T}_{\epsilon}[\right.$.

With our hypotheses, the estimate (A.8) gives

$$
\left\|U_{\epsilon}(t)\right\|_{B_{2,1}^{\frac{11}{4}} \lesssim C_{0} \epsilon^{-\alpha} e^{C\left(C_{0} t+V_{\epsilon}(t)\right)}}
$$

and

$$
\left\|U_{\epsilon}(t)\right\|_{B_{2, q}^{s+\frac{11}{4}}} \lesssim C_{0} \epsilon^{-\beta} e^{C\left(C_{0} t+V_{\epsilon}(t)\right)} .
$$

Using (4.5) and (4.6) in the dispersive inequalities (B.4), (B.5) and (B.6) gives

$$
\left\|P_{ \pm 1} U_{\epsilon}\right\|_{L_{t}^{1}(\mathrm{Lip})} \lesssim g_{\epsilon}^{(0)}(t) \stackrel{\text { def }}{=} C_{0} t^{\frac{3}{4}} \epsilon^{\frac{1}{4}-\alpha} e^{C\left(C_{0} t+V_{\epsilon}(t)\right)}
$$

and

$$
\left\|P_{ \pm 1} U_{\epsilon}\right\|_{L_{t}^{1}\left(C^{s+1}\right)} \lesssim g_{\epsilon}^{(s)}(t) \stackrel{\text { def }}{=} C_{0} t^{\frac{3}{4}} \epsilon^{\frac{1}{4}-\beta} e^{C\left(C_{0} t+V_{\epsilon}(t)\right)} .
$$

Finally, using (4.7) in the estimation on the curl (3.5) and in the energy estimate

$$
\begin{aligned}
& \left\|U_{\epsilon}(t)\right\|_{L^{2}} \leq\left\|U_{0, \epsilon}\right\|_{L^{2}}+\left\|\sigma^{\prime} \cdot \nabla \sigma\right\|_{L^{2}} t \\
& +\int_{0}^{t}\left(\frac{1}{2}\left\|\operatorname{div} U_{\epsilon}^{\prime}\left(t^{\prime}\right)\right\|_{L^{\infty}}+\bar{\gamma}\left\|\nabla U_{\epsilon}^{3}\left(t^{\prime}\right)\right\|_{L^{\infty}}+\|\nabla \sigma\|_{L^{\infty}}\right)\left\|U_{\epsilon}\left(t^{\prime}\right)\right\|_{L^{2}} d t^{\prime}
\end{aligned}
$$

gives

$$
\left\|\Omega_{\epsilon}(t)\right\|_{L^{\infty}} \lesssim C_{0} e^{C g_{\epsilon}^{(0)}(t)}
$$

and

$$
\left\|U_{\epsilon}(t)\right\|_{L^{2}} \lesssim C_{0} e^{C\left(C_{0} t+g_{\epsilon}^{(0)}(t)\right)}
$$

So

$$
\left\|P_{0} U_{\epsilon}(t)\right\|_{\text {Lip }} \lesssim C_{0} e^{C\left(C_{0} t+g_{\epsilon}^{(0)}(t)\right)}+C_{0} e^{C g_{\epsilon}^{(0)}(t)} \ln \left(e+X_{\epsilon}(t)\right) .
$$


Now let's estimate $X_{\epsilon}(t)$. First, (4.4) yields

$$
\left\|\left[W_{\epsilon}(t)\right]^{-1}\right\|_{L^{\infty}} \lesssim C_{0} e^{C\left(C_{0} t+V_{\epsilon}(t)\right)} .
$$

Second,

$$
\begin{aligned}
\left\|w_{\epsilon}^{\nu}(t)\right\|_{C^{s}} \lesssim & \left\|w_{0}^{\nu}\right\|_{C^{s}}+\int_{0}^{t}\left\|U_{\epsilon}^{\prime}\left(t^{\prime}\right)+\sigma^{\prime}\right\|_{\text {Lip }}\left\|w_{\epsilon}^{\nu}\left(t^{\prime}\right)\right\|_{C^{s}} d t^{\prime} \\
& +\int_{0}^{t}\left\|w_{\epsilon}^{\nu}\left(t^{\prime}\right) \cdot \nabla\left(U_{\epsilon}^{\prime}\left(t^{\prime}\right)+\sigma^{\prime}\right)\right\|_{C^{s}} d t^{\prime} \\
\lesssim & C_{0}+C_{0} \int_{0}^{t}\left\|w_{\epsilon}^{\nu}\left(t^{\prime}\right)\right\|_{C^{s}} d t^{\prime} \\
& +\int_{0}^{t}\left(\left\|P_{0} U_{\epsilon}\left(t^{\prime}\right)\right\|_{\text {Lip }}+\left\|\left(P_{1}+P_{-1}\right) U_{\epsilon}\left(t^{\prime}\right)\right\|_{\text {Lip }}\right)\left\|w_{\epsilon}^{\nu}\left(t^{\prime}\right)\right\|_{C^{s}} d t^{\prime} \\
& +\int_{0}^{t}\left\|w_{\epsilon}^{\nu}\left(t^{\prime}\right)\right\|_{L^{\infty}}\left\|\left(P_{1}+P_{-1}\right) U_{\epsilon}\left(t^{\prime}\right)\right\|_{C^{s+1}} d t^{\prime} \\
& +\int_{0}^{t}\left\|\operatorname{div}\left(w_{\epsilon}^{\nu}\left(t^{\prime}\right) \Omega_{\epsilon}\left(t^{\prime}\right)\right)\right\|_{C^{s-1}} d t^{\prime}
\end{aligned}
$$

because

$$
\left\|w_{\epsilon}^{\nu} \cdot \nabla P_{0} U_{\epsilon}\right\|_{C^{s}} \lesssim\left\|w_{\epsilon}^{\nu}\right\|_{C^{s}}\left(C_{0}+\left\|P_{0} U_{\epsilon}\right\|_{\operatorname{Lip}}\right)+\left\|\operatorname{div}\left(w_{\epsilon}^{\nu} \Omega_{\epsilon}\right)\right\|_{C^{s-1}},
$$

the field $\tilde{v}_{\epsilon}$ being more regular in the same directions as his curl $[10$, Section 2.4.1]. Third, as a consequence of (C.1) and (4.3),

$$
\left\{\begin{array}{l}
\partial_{t} \operatorname{div}\left(w_{\epsilon}^{\nu} \Omega_{\epsilon}\right)+\operatorname{div}\left(\left(U_{\epsilon}^{\prime}+\sigma^{\prime}\right) \operatorname{div}\left(w_{\epsilon}^{\nu} \Omega_{\epsilon}\right)\right)=0 \\
\left.\operatorname{div}\left(w_{\epsilon}^{\nu} \Omega_{\epsilon}\right)\right|_{t=0}=\operatorname{div}\left(w_{0}^{\nu} \Omega_{0, \epsilon}\right) .
\end{array}\right.
$$

Hence, using an a priori estimate for Hölder spaces of negative index [3, Lemma 4.1.1],

$$
\begin{aligned}
& \left\|\operatorname{div}\left(w_{\epsilon}^{\nu}(t) \Omega_{\epsilon}(t)\right)\right\|_{C^{s-1}} \\
& \lesssim\left\|\operatorname{div}\left(w_{0}^{\nu} \Omega_{0, \epsilon}\right)\right\|_{C^{s-1}}+\int_{0}^{t}\left\|\operatorname{div} U_{\epsilon}^{\prime}\left(t^{\prime}\right)\right\|_{L^{\infty}}\left\|\operatorname{div}\left(w_{\epsilon}^{\nu}\left(t^{\prime}\right) \Omega_{\epsilon}\left(t^{\prime}\right)\right)\right\|_{C^{s-1}} d t^{\prime} \\
& \quad+\int_{0}^{t}\left\|U_{\epsilon}^{\prime}+\sigma^{\prime}\right\|_{\operatorname{Lip}}\left\|\operatorname{div}\left(w_{\epsilon}^{\nu}\left(t^{\prime}\right) \Omega_{\epsilon}\left(t^{\prime}\right)\right)\right\|_{C^{s-1}} d t^{\prime} \\
& \quad+\int_{0}^{t}\left\|\operatorname{div} U_{\epsilon}^{\prime}\left(t^{\prime}\right)\right\|_{C^{s}}\left\|w_{\epsilon}^{\nu}\left(t^{\prime}\right) \Omega_{\epsilon}\left(t^{\prime}\right)\right\|_{L^{\infty}} d t^{\prime} \\
& \lesssim C_{\epsilon}+\int_{0}^{t}\left(C_{0}+\left\|U_{\epsilon}\left(t^{\prime}\right)\right\|_{\operatorname{Lip}}\right)\left\|\operatorname{div}\left(w_{\epsilon}^{\nu}\left(t^{\prime}\right) \Omega_{\epsilon}\left(t^{\prime}\right)\right)\right\|_{C^{s-1}} d t^{\prime} \\
& \quad+C_{0}^{2} e^{C\left(C_{0} t+V_{\epsilon}(t)\right)} \int_{0}^{t}\left\|\left(P_{1}+P_{-1}\right) U_{\epsilon}\left(t^{\prime}\right)\right\|_{C^{s+1}} d t^{\prime}
\end{aligned}
$$




$$
\begin{aligned}
\left\|\operatorname{div}\left(w_{\epsilon}^{\nu}(t) \Omega_{\epsilon}(t)\right)\right\|_{C^{s-1}} & \lesssim\left(C_{\epsilon}+C_{0}^{2} e^{C\left(C_{0} t+V_{\epsilon}(t)\right)} g_{\epsilon}^{(s)}(t)\right) e^{C\left(C_{0} t+V_{\epsilon}(t)\right)} \\
& \lesssim C_{\epsilon} e^{C\left(C_{0} t+V_{\epsilon}(t)\right)}+C_{0}^{2} g_{\epsilon}^{(s)}(t)
\end{aligned}
$$

Substituting this inequality in (4.10), we get

$$
\left\|w_{\epsilon}^{\nu}(t)\right\|_{C^{s}} \lesssim\left(C_{\epsilon}+C_{0}+C_{0} g_{\epsilon}^{(s)}(t)\right) e^{C\left(C_{0} t+\int_{0}^{t}\left\|P_{0} U_{\epsilon}\left(t^{\prime}\right)\right\|_{\text {Lip }} d t^{\prime}+g_{\epsilon}^{(0)}(t)\right)}
$$

So

$$
X_{\epsilon}(t) \lesssim\left(C_{\epsilon}+C_{0}+C_{0}^{2} g_{\epsilon}^{(s)}(t)\right) e^{C\left(C_{0} t+\int_{0}^{t}\left\|P_{0} U_{\epsilon}\left(t^{\prime}\right)\right\|_{\text {Lip }} d t^{\prime}+g_{\epsilon}^{(0)}(t)\right)} .
$$

Thus

$$
\begin{aligned}
& \left\|P_{0} U_{\epsilon}(t)\right\|_{\text {Lip }} \\
& \lesssim C_{0} e^{C\left(C_{0} t+g_{\epsilon}^{(0)}(t)\right)}+C_{0} e^{C g_{\epsilon}^{(0)}(t)}\left(\ln \left(e+C_{\epsilon}+C_{0}+C_{0}^{2} g_{\epsilon}^{(s)}(t)\right)\right. \\
& \left.+C_{0} t+\int_{0}^{t}\left\|P_{0} U_{\epsilon}\left(t^{\prime}\right)\right\|_{\text {Lip }} d t^{\prime}+g_{\epsilon}^{(0)}(t)\right) \\
& \lesssim C_{0} e^{C\left(C_{0} t+g_{\epsilon}^{(0)}(t)\right)}+C_{0} e^{C g_{\epsilon}^{(0)}(t)}\left(\ln \left(e+C_{\epsilon}+C_{0}+C_{0}^{3} t^{\frac{3}{4}} \epsilon^{\frac{1}{4}-\beta}\right)\right. \\
& \left.+C_{0} t+\int_{0}^{t}\left\|P_{0} U_{\epsilon}\left(t^{\prime}\right)\right\|_{\text {Lip }} d t^{\prime}+g_{\epsilon}^{(0)}(t)\right) \\
& \lesssim C_{0} e^{C\left(C_{0} t+g_{\epsilon}^{(0)}(t)\right)}+C_{0} e^{C g_{\epsilon}^{(0)}(t)}\left(\ln \left(e+C_{\epsilon}+2 C_{0}\right)+C_{0} t\right) \\
& +C_{0} e^{C g_{\epsilon}^{(0)}(t)} \int_{0}^{t}\left\|P_{0} U_{\epsilon}\left(t^{\prime}\right)\right\|_{\text {Lip }} d t^{\prime}
\end{aligned}
$$

if $C_{0}^{2} t^{\frac{3}{4}} \epsilon^{\frac{1}{4}-\beta} \leq 1$. Therefore,

$$
\left\|P_{0} U_{\epsilon}(t)\right\|_{\operatorname{Lip}} \lesssim C_{0} e^{C\left(C_{0} t+g_{\epsilon}^{(0)}(t)\right)}\left(\ln \left(e+C_{\epsilon}+2 C_{0}\right)\right) e^{C C_{0} t e^{C g_{\epsilon}^{(0)}(t)}},
$$

and finally

$$
V_{\epsilon}(t) \lesssim e^{C C_{0}(t+1) e^{C g_{\epsilon}^{(0)}(t)}} \ln \left(e+C_{\epsilon}\right),
$$

for all $t \in\left[0, T_{\epsilon}^{(\mu)}\right] \cap\left[0, T_{\epsilon}[\right.$.

From this we conclude that, on $\left[0, T_{\epsilon}^{(\mu)}\right] \cap\left[0, T_{\epsilon}\left[\right.\right.$, the inequality $g_{\epsilon}^{(0)}(t) \leq 1$ implies

$$
\begin{aligned}
g_{\epsilon}^{(0)}(t) & \leq \epsilon^{\frac{1}{4}-\alpha} e^{C C_{0} t}\left(e+C_{\epsilon}\right)^{e^{C C_{0}(t+1) e^{C g_{\epsilon}^{(0)}(t)}}} \\
& \leq \epsilon^{\frac{1}{4}-\alpha}\left(e+C_{\epsilon}\right)^{C C_{0}(t+1)} \\
& \leq \epsilon^{\mu}
\end{aligned}
$$

Hence (4.1) and (4.2) are proved with $T_{\epsilon}^{(\mu)}$ replaced by $\min \left(T_{\epsilon}^{(\mu)}, T_{\epsilon}\right)$, which in turn proves that $T_{\epsilon}>T_{\epsilon}^{(\mu)}$. 
Theorem 4. In addition to the hypotheses of Theorem 3, suppose that $P_{0} U_{0, \epsilon} \rightarrow U_{0}$ in $L^{2}\left(\mathbb{R}^{2}\right)$, that $\left\|\Omega_{0, \epsilon}\right\|_{L^{a}} \leq C_{0}$ for some $a<\infty$, that

$$
\ln \left(\epsilon^{-1}\right) / \ln \left(e+C_{\epsilon}\right) \rightarrow+\infty \text { as } \epsilon \rightarrow 0,
$$

and that

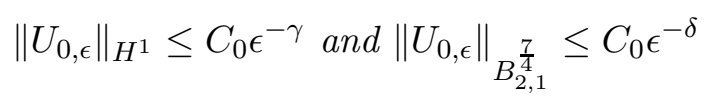

with $\gamma \leq \delta \leq \alpha$ and

$$
\gamma+\delta<\frac{1}{4}
$$

Then, for any stationary field $\sigma^{\prime}$ defined by (1.5), $\sigma^{\prime}+\left(P_{0} U_{\epsilon}\right)^{\prime}$ converges in $L_{\mathrm{loc}}^{\infty}\left(\mathbb{R}^{+} ; \sigma^{\prime}+L^{2}\right)$ to the unique solution $v \in C\left(\mathbb{R}^{+} ; \sigma^{\prime}+L^{2}\right)$ of the incompressible Euler system

$$
\left\{\begin{array}{l}
\partial_{t} v+v \cdot \nabla v=-\nabla p \\
\operatorname{div} v=0 \\
\left.v\right|_{t=0}=\sigma^{\prime}+U_{0}^{\prime}
\end{array}\right.
$$

such that $p \in L_{\mathrm{loc}}^{\infty}\left(\mathbb{R}^{+} ; L^{2}\right)$ and $\operatorname{rot} v \in L^{\infty}\left(\mathbb{R}^{+} ; L^{\infty} \cap L^{a}\right)$.

Proof. Let $T>0$ be fixed. Choose $\mu>\gamma+\delta-\alpha$; by Theorem 3 and our hypothesis on $C_{\epsilon}$, there is a $\epsilon>0$ such that $T_{\epsilon}^{(\mu)} \geq T$. Now the proof can proceed exactly as the end of Theorem 1's proof, except for the estimates

$$
\begin{aligned}
\|Q U\|_{L_{T}^{1}\left(L^{\infty}\right)}\left\|\nabla\left(U_{2}(t)+\sigma\right)\right\|_{L_{T}^{\infty}\left(L^{2}\right)} & \lesssim \epsilon^{\frac{1}{4}-\delta} e^{C\left(C_{0}(T+1)+V_{\epsilon}(T)\right)} \epsilon^{-\gamma} \\
& \lesssim \epsilon^{\alpha+\mu-\gamma-\delta}
\end{aligned}
$$

and

$$
\begin{aligned}
\|\nabla Q U\|_{L_{T}^{1}\left(L^{p}\right)} & \lesssim \epsilon^{\frac{1}{4}-\alpha-\frac{1}{2 p}} e^{C\left(C_{0}(T+1)+V_{\epsilon}(T)\right)} \\
& \lesssim \epsilon^{\mu-\frac{1}{2 p}}
\end{aligned}
$$

for all $p>2$, and for the choice of $b$ in (3.8) $\left(b=a-\ln J_{\eta}\right)$.

\section{A Energy estimates}

As (2.1) is a very simple example of quasi-linear symmetric hyperbolic system, energy estimates are also very easy to prove. The main point is that terms in $\epsilon^{-1}$ disappear in the integration by parts, leaving estimates uniform with respect to $\epsilon$.

Lemma 1. If $F, G$ and $V={ }^{t}\left(V^{\prime}, V^{3}\right)$ are solutions of

$$
\left\{\begin{array}{l}
\partial_{t} F+V^{\prime} \cdot \nabla F+\left(V^{3}+\frac{1}{\epsilon}\right) B(D) F=G \\
\left.F\right|_{t=0}=F_{0}
\end{array}\right.
$$


then

$$
\begin{aligned}
\|F(t)\|_{L^{2}} \leq & \left\|F_{0}\right\|_{L^{2}}+\int_{0}^{t}\left\|G\left(t^{\prime}\right)\right\|_{L^{2}} d t^{\prime} \\
& +\int_{0}^{t}\left(\frac{1}{2}\left\|\operatorname{div} V^{\prime}\left(t^{\prime}\right)\right\|_{L^{\infty}}+\left\|\nabla V^{3}\left(t^{\prime}\right)\right\|_{L^{\infty}}\right)\left\|F\left(t^{\prime}\right)\right\|_{L^{2}} d t^{\prime} .
\end{aligned}
$$

Proof. Multiplying both sides of (A.1) by $F(t)$ gives

$$
\frac{1}{2} \partial_{t}|F|^{2}+\frac{1}{2} V^{\prime} \cdot \nabla|F|^{2}+\left(V^{3}+\frac{1}{\epsilon}\right) \operatorname{div}\left(F^{3} F^{\prime}\right)=G \cdot F,
$$

because $(B(D) F) \cdot F=\partial_{1}\left(F^{3} F^{1}\right)+\partial_{2}\left(F^{3} F^{2}\right)$. Hence, after integration by parts,

$$
\begin{aligned}
\frac{1}{2} \partial_{t} \int_{\mathbb{R}^{2}} \mid F( & t, x)\left.\right|^{2} d x-\frac{1}{2} \int_{\mathbb{R}^{2}}|F(t, x)|^{2} \operatorname{div} V^{\prime}(t, x) d x \\
- & \int_{\mathbb{R}^{2}} F^{3}(t, x) F^{\prime}(t, x) \cdot \nabla V^{3}(t, x) d x=\int_{\mathbb{R}^{2}} G(t, x) \cdot F(t, x) d x
\end{aligned}
$$

and thus

$$
\begin{aligned}
\frac{1}{2} \partial_{t}\|F(t)\|_{L^{2}}^{2} \leq & \|G(t)\|_{L^{2}}\|F(t)\|_{L^{2}}+\frac{1}{2}\|F(t)\|_{L^{2}}^{2}\left\|\operatorname{div} V^{\prime}(t)\right\|_{L^{\infty}} \\
& +\left\|F^{3}(t)\right\|_{L^{2}}\left\|F^{\prime}(t)\right\|_{L^{2}}\left\|\nabla V^{3}(t)\right\|_{L^{\infty}}
\end{aligned}
$$

from which (A.2) follows, by Gronwall's lemma.

As a consequence of Lemma 1, taking $F=U_{\epsilon}, V={ }^{t}\left(U_{\epsilon}^{\prime}+\sigma^{\prime}, \bar{\gamma} U_{\epsilon}^{3}\right)$ and $G=-\left(U_{\epsilon}^{\prime}+\sigma^{\prime}\right) \cdot \nabla \sigma$, we get the estimate (4.9).

Using a dyadic decomposition in frequency [3, 25], we also deduce from Lemma 1 uniform estimates for higher norms. We will need the operators of frequency truncation $\Delta_{m}$, defined for $m \geq-1$ by

$$
\Delta_{m} u= \begin{cases}\varphi\left(2^{-m} D\right) u & \text { if } m \geq 0, \\ \chi(D) u & \text { if } m=-1,\end{cases}
$$

where $\chi$ is a smooth, compactly supported function, equal to 1 near the origin, and

$$
\varphi(\xi)=\chi(\xi / 2)-\chi(\xi) .
$$

We also set $\Delta_{m}=0$ for all $m<-1$, and

$$
S_{n} \stackrel{\text { def }}{=} \chi\left(2^{-n} D\right)=\sum_{m \leq n-1} \Delta_{m}
$$

for all $n \geq 0$. Recall that Besov spaces are defined [25, Section 2.3] by

$$
B_{p, q}^{s}\left(\mathbb{R}^{d}\right)=\left\{f \in \mathcal{S}^{\prime}\left(\mathbb{R}^{d}\right) ;\|f\|_{B_{p, q}^{s}\left(\mathbb{R}^{d}\right)} \stackrel{\text { def }}{=}\left\|\left(2^{m s}\left\|\Delta_{m} f\right\|_{L^{p}}\right)_{m=-1}^{+\infty}\right\|_{l^{q}}<\infty\right\}
$$

and that Sobolev spaces constructed on $L^{2}$ are special cases of Besov spaces:

$$
H^{s} \simeq B_{2,2}^{s} .
$$


Lemma 2. Let $F, G$ and $V$ as in Lemma 1. Let also $r>0$ and $q \in[1,+\infty]$. Then

$$
\begin{aligned}
& \|F(t)\|_{B_{2, q}^{r}} \lesssim\left\|F_{0}\right\|_{B_{2, q}^{r}}+\int_{0}^{t}\left\|G\left(t^{\prime}\right)\right\|_{B_{2, q}^{r}} d t^{\prime} \\
& \quad+\int_{0}^{t}\left\|V\left(t^{\prime}\right)\right\|_{\text {Lip }}\left\|F\left(t^{\prime}\right)\right\|_{B_{2, q}^{r}} d t^{\prime}+\int_{0}^{t}\left\|F\left(t^{\prime}\right)\right\|_{\text {Lip }}\left\|\nabla V\left(t^{\prime}\right)\right\|_{B_{2, q}^{r-1}} d t^{\prime} .
\end{aligned}
$$

Proof. For all integers $m \geq-1$, we have

$$
\begin{aligned}
\partial_{t} \Delta_{m} F+V^{\prime} \cdot \nabla \Delta_{m} F+\left(V^{3}\right. & \left.+\frac{1}{\epsilon}\right) B(D) \Delta_{m} F \\
& =\Delta_{m} G+\left[V^{\prime} \cdot \nabla, \Delta_{m}\right] F+\left[V^{3} B(D), \Delta_{m}\right] F .
\end{aligned}
$$

Lemma 1 gives

$$
\begin{aligned}
\left\|\Delta_{m} F(t)\right\|_{L^{2}} \leq & \left\|\Delta_{m} F_{0}\right\|_{L^{2}}+\int_{0}^{t}\left\|\Delta_{m} G\left(t^{\prime}\right)\right\|_{L^{2}} d t^{\prime} \\
+\int_{0}^{t} \|\left[V^{\prime}\left(t^{\prime}\right)\right. & \left.\cdot \nabla, \Delta_{m}\right] F\left(t^{\prime}\right)\left\|_{L^{2}} d t^{\prime}+\int_{0}^{t}\right\|\left[V^{3}\left(t^{\prime}\right) B(D), \Delta_{m}\right] F\left(t^{\prime}\right) \|_{L^{2}} d t^{\prime} \\
& +\int_{0}^{t}\left(\frac{1}{2}\left\|\operatorname{div} V^{\prime}\left(t^{\prime}\right)\right\|_{L^{\infty}}+\left\|\nabla V^{3}\left(t^{\prime}\right)\right\|_{L^{\infty}}\right)\left\|\Delta_{m} F\left(t^{\prime}\right)\right\|_{L^{2}} d t^{\prime}
\end{aligned}
$$

so

$$
\begin{gathered}
\|F(t)\|_{B_{2, q}^{r}}=\left\|\left(2^{m r}\left\|\Delta_{m} F(t)\right\|_{L^{2}}\right)_{m=-1}^{+\infty}\right\|_{l^{q}} \\
\lesssim\left\|\left(2^{m r}\left\|\Delta_{m} F_{0}\right\|_{L^{2}}\right)_{m=-1}^{+\infty}\right\|_{l^{q}}+\int_{0}^{t}\left\|\left(2^{m r}\left\|G\left(t^{\prime}\right)\right\|_{L^{2}}\right)_{m=-1}^{+\infty}\right\|_{l^{q}} d t^{\prime} \\
\quad+\int_{0}^{t}\left\|\left(2^{m r}\left\|\left[V^{\prime}\left(t^{\prime}\right) \cdot \nabla, \Delta_{m}\right] F\left(t^{\prime}\right)\right\|_{L^{2}}\right)_{m=-1}^{+\infty}\right\|_{l^{q}} d t^{\prime} \\
\quad+\int_{0}^{t}\left\|\left(2^{m r}\left\|\left[V^{3}\left(t^{\prime}\right) B(D), \Delta_{m}\right] F\left(t^{\prime}\right)\right\|_{L^{2}}\right)_{m=-1}^{+\infty}\right\|_{l^{q}} d t^{\prime} \\
+\int_{0}^{t}\left(\frac{1}{2}\left\|\operatorname{div} V^{\prime}\left(t^{\prime}\right)\right\|_{L^{\infty}}+\left\|\nabla V^{3}\left(t^{\prime}\right)\right\|_{L^{\infty}}\right)\left\|\left(2^{m r}\left\|\Delta_{m} F\left(t^{\prime}\right)\right\|_{L^{2}}\right)_{m=-1}^{+\infty}\right\|_{l^{q}} d t^{\prime},
\end{gathered}
$$

which can be bounded by the right member of (A.4), thanks to the commutator estimate

$$
\left\|\left(2^{m r}\left\|\left[a \cdot \nabla, \Delta_{m}\right] b\right\|_{L^{2}}\right)_{m=-1}^{+\infty}\right\|_{l^{q}} \lesssim\|a\|_{\text {Lip }}\|b\|_{B_{2, q}^{r}}+\|b\|_{\text {Lip }}\|\nabla a\|_{B_{2, q}^{r-1}}
$$

The proof of (A.5) will be included for the convenience of the reader, although the proof of similar estimates can be found elsewhere [3, p. 67-70], 
[12, p. 23-25], [9, p. 22-23], [11, p. 182-183]. The commutator is decomposed following the method of Bony:

$$
\begin{aligned}
{\left[a \cdot \nabla, \Delta_{m}\right] b=} & \sum_{m^{\prime}=m-N}^{m+N}\left[\left(S_{m^{\prime}-1} a\right) \cdot \nabla, \Delta_{m}\right] \Delta_{m^{\prime}} b \\
& +c_{m}+\sum_{m^{\prime}=\min (0, m-N)}^{+\infty}\left[\left(\Delta_{m^{\prime}} a\right) \cdot \nabla, \Delta_{m}\right] S_{m^{\prime}+2} b,
\end{aligned}
$$

with

$$
c_{m} \stackrel{\text { def }}{=} \begin{cases}{\left[\left(\Delta_{-1} a\right) \cdot \nabla, \Delta_{m}\right] S_{1} b} & \text { if } m-N \leq-1, \\ 0 & \text { otherwise. }\end{cases}
$$

The integer $N$ depends only on the function $\chi$ chosen for the dyadic decomposition. On one hand, we have

$$
\left\|\left(2^{m r}\left\|c_{m}\right\|_{L^{2}}\right)_{m=-1}^{+\infty}\right\|_{l^{2}} \lesssim\|\nabla a\|_{L^{\infty}}\|b\|_{L^{2}}
$$

and

$$
\begin{aligned}
\|\left(2^{m r} \|\right. & \left.\sum_{m^{\prime}=m-N}^{m+N}\left[\left(S_{m^{\prime}-1} a\right) \cdot \nabla, \Delta_{m}\right] \Delta_{m^{\prime}} b \|_{L^{2}}\right)_{m=-1}^{+\infty} \|_{l^{q}} \\
& \lesssim\left\|\left(2^{m r} \sum_{m^{\prime}=m-N}^{m+N} 2^{-m}\left\|\nabla S_{m^{\prime}-1} a\right\|_{L^{\infty}}\left\|\nabla \Delta_{m^{\prime}} b\right\|_{L^{2}}\right)_{m=-1}^{+\infty}\right\|_{l^{q}} \\
& \lesssim\|\nabla a\|_{L^{\infty}}\left\|\left(\sum_{m^{\prime}=m-N}^{m+N} 2^{\left(m-m^{\prime}\right)(r-1)} 2^{m^{\prime}(r-1)}\left\|\Delta_{m^{\prime}} \nabla b\right\|_{L^{2}}\right)_{m=-1}^{+\infty}\right\|_{l^{q}} \\
& \lesssim\|\nabla a\|_{L^{\infty}}\|\nabla b\|_{B_{2, q}^{r-1}},
\end{aligned}
$$

because of the inequality

$$
\left\|\left[f, \Delta_{m}\right] g\right\|_{L^{2}} \lesssim 2^{-m}\|\nabla f\|_{L^{\infty}}\|g\|_{L^{2}},
$$

valid for $f \in \operatorname{Lip}, g \in L^{2}$ and $m \geq-1$. On the other hand, we may write

$$
\begin{aligned}
\|\left(2^{m r} \|\right. & \left.\sum_{m^{\prime}=\min (0, m-N)}^{+\infty}\left[\left(\Delta_{m^{\prime}} a\right) \cdot \nabla, \Delta_{m}\right] S_{m^{\prime}+2} b \|_{L^{2}}\right)_{m=-1}^{+\infty} \|_{l^{q}} \\
& \lesssim \|\left(2^{m r} \sum_{m^{\prime}=\min (0, m-N)}^{+\infty}\left\|\Delta_{m^{\prime}} a\right\|_{L^{2}}\left\|S_{m^{\prime}+2} \nabla b\right\|_{\left.L^{\infty}\right)_{m=-1}^{+\infty} \|_{l^{q}}}\right. \\
& \lesssim \|\left(\sum_{m^{\prime}=\min (0, m-N)}^{+\infty} 2^{\left(m-m^{\prime}\right) r} 2^{m^{\prime}(r-1)}\left\|\Delta_{m^{\prime}} \nabla a\right\|_{L^{2}}\|\nabla b\|_{\left.L^{\infty}\right)_{m=-1}^{+\infty} \|_{l^{q}}}\right. \\
& \lesssim\|\nabla b\|_{L^{\infty}}\|\nabla a\|_{B_{2, q}^{r-1}},
\end{aligned}
$$


thanks to Bernstein's inequalities [3, Lemma 2.1.1]

$$
\left\|\Delta_{m^{\prime}} a\right\|_{L^{2}} \lesssim 2^{-m^{\prime}}\left\|\Delta_{m^{\prime}} \nabla a\right\|_{L^{2}},
$$

for all $m^{\prime} \geq 0$.

As a consequence of Lemma 2 we have, for all $r>0$ and $q \in[1, \infty]$, the a priori estimates

$$
\begin{aligned}
\left\|U_{\epsilon}(t)\right\|_{B_{2, q}^{r}} \lesssim\left\|U_{0, \epsilon}\right\|_{B_{2, q}^{r}}+ & C_{0} t+C_{0} \int_{0}^{t}\left\|U_{\epsilon}\left(t^{\prime}\right)\right\|_{\text {Lip }} d t^{\prime} \\
& +\int_{0}^{t}\left(C_{0}+\left\|U_{\epsilon}\left(t^{\prime}\right)\right\|_{\text {Lip }}\right)\left\|U_{\epsilon}\left(t^{\prime}\right)\right\|_{B_{2, q}^{r}} d t^{\prime},
\end{aligned}
$$

provided $\|\sigma\|_{\text {Lip }}+\left\|\sigma^{\prime} \cdot \nabla \sigma\right\|_{B_{2, q}^{r}}+\|\nabla \sigma\|_{B_{2, q}^{r}} \leq C_{0}$.

\section{B Dispersive estimates}

Since

$$
\left\{\begin{array}{l}
\partial_{t} P_{ \pm 1} U_{\epsilon} \pm \frac{i}{\epsilon}|D| P_{ \pm 1} U_{\epsilon}=-P_{ \pm 1} I_{\epsilon} \\
\left.\left(P_{ \pm 1} U_{\epsilon}\right)\right|_{t=0}=P_{ \pm 1} U_{0, \epsilon}
\end{array}\right.
$$

with

$$
I_{\epsilon}=\left(U_{\epsilon}^{\prime}+\sigma^{\prime}\right) \cdot \nabla\left(U_{\epsilon}+\sigma\right)+\bar{\gamma} U_{\epsilon}^{3} B(D) U_{\epsilon},
$$

we have for $P_{ \pm 1} U_{\epsilon}$ Strichartz estimates similar to those valid for solutions of either half of the wave equation,

$$
\left\{\begin{array}{l}
\partial_{t} f \pm \frac{i}{\epsilon}|D| f=g \\
\left.f\right|_{t=0}=f_{0}
\end{array}\right.
$$

Lemma 3. Let $s \in] 0,1\left[\right.$ and $q \in[1,4]$. If $f, f_{0}$ and $g$ satisfy (B.1), then

$$
\|f\|_{L_{t}^{r}\left(L^{p}\right)} \lesssim \epsilon^{\frac{1}{4}-\frac{1}{2 p}}\left(\left\|f_{0}\right\|_{B_{2,1}^{\frac{3}{4}}-\frac{3}{2 p}}+\int_{0}^{t}\left\|g\left(t^{\prime}\right)\right\|_{B_{2,1}^{\frac{3}{4}-\frac{3}{2 p}}} d t^{\prime}\right)
$$

for all $2 \leq p \leq+\infty$, with $r \stackrel{\text { def }}{=} 4+8 /(p-2)$, and

$$
\|f\|_{L_{t}^{4}\left(C^{s+1}\right)} \lesssim \epsilon^{\frac{1}{4}}\left(\left\|f_{0}\right\|_{B_{2, q}^{s+\frac{7}{4}}}+\int_{0}^{t}\left\|g\left(t^{\prime}\right)\right\|_{B_{2, q}^{s+\frac{11}{4}}} d t^{\prime}\right) .
$$

We will actually use three estimates following from Lemma 3:

$$
\begin{aligned}
& \left\|P_{ \pm 1} U_{\epsilon}\right\|_{L_{t}^{4}\left(L^{\infty}\right)} \lesssim \epsilon^{\frac{1}{4}}\left(\left\|P_{ \pm 1} U_{0, \epsilon}\right\|_{B_{2,1}^{\frac{3}{4}}}\right. \\
& \left.\quad+\int_{0}^{t}\left(C_{0}+C_{0}\left\|U_{\epsilon}\left(t^{\prime}\right)\right\|_{B_{2,1}^{\frac{7}{4}}}+\left\|U_{\epsilon}\left(t^{\prime}\right)\right\|_{\text {Lip }}\left\|U_{\epsilon}\left(t^{\prime}\right)\right\|_{B_{2,1}^{\frac{7}{4}}}\right) d t^{\prime}\right)
\end{aligned}
$$




$$
\begin{aligned}
\| & \nabla P_{ \pm 1} U_{\epsilon} \|_{L_{t}^{4+\frac{8}{p-2}}\left(L^{p}\right)} \lesssim \epsilon^{\frac{1}{4}-\frac{1}{2 p}}\left(\left\|P_{ \pm 1} U_{0, \epsilon}\right\|_{B_{2,1}^{\frac{7}{4}-\frac{3}{2 p}}}\right. \\
& \left.+\int_{0}^{t}\left(C_{0}+C_{0}\left\|U_{\epsilon}\left(t^{\prime}\right)\right\|_{B_{2,1}^{\frac{11}{4}-\frac{3}{2 p}}}+\left\|U_{\epsilon}\left(t^{\prime}\right)\right\|_{\text {Lip }}\left\|U_{\epsilon}\left(t^{\prime}\right)\right\|_{B_{2,1}^{\frac{11}{4}-\frac{3}{2 p}}}\right) d t^{\prime}\right)
\end{aligned}
$$

for $2<p \leq+\infty$, and

$$
\begin{aligned}
& \left\|P_{ \pm 1} U_{\epsilon}\right\|_{L_{t}^{4}\left(C^{s+1}\right)} \lesssim \epsilon^{\frac{1}{4}}\left(\left\|P_{ \pm 1} U_{0, \epsilon}\right\|_{B_{2, q}^{s+\frac{7}{4}}}\right. \\
& \left.\quad+\int_{0}^{t}\left(C_{0}+C_{0}\left\|U_{\epsilon}\left(t^{\prime}\right)\right\|_{B_{2, q}^{s+\frac{11}{4}}}+\left\|U_{\epsilon}\left(t^{\prime}\right)\right\|_{\mathrm{Lip}}\left\|U_{\epsilon}\left(t^{\prime}\right)\right\|_{B_{2, q}^{s+\frac{11}{4}}}\right) d t^{\prime}\right)
\end{aligned}
$$

for $q \in[1,4]$ and $s \in] 0,1[$; these are true provided

$$
\left\|\sigma^{\prime} \cdot \nabla \sigma\right\|_{B_{2, q}^{s+\frac{7}{4}}}+\|\nabla \sigma\|_{B_{2, q}^{s+\frac{11}{4}}}+\|\sigma\|_{C^{s+\frac{11}{4}}} \leq C_{0} .
$$

Proof of Lemma 3. We give a proof of (B.2) and (B.3), but let us stress once again that these are just the classical, well-known Strichartz estimates for the wave equation [15], which were already used, in order to solve a problem of incompressible limit, by Ukai [26].

This proof is directly inspired by the study of dispersion in rotating fluids due to Chemin, Desjardins, Gallagher and Grenier [4,5]. A proof is also sketched in a recent paper by Danchin [6, Proposition 7.1].

We may assume that $\epsilon=1$, because setting $\tilde{f}(t, x)=f(\epsilon t, x)$ and $\tilde{g}(t, x)=\epsilon g(\epsilon t, x)$ will give (B.2) and (B.3) for all other $\epsilon$.

The first step is a dispersive inequality for solutions of (B.1) whose Fourier transform is supported in a fixed annulus of $\mathbb{R}^{2}$ : if $\psi: \mathbb{R}^{2} \rightarrow \mathbb{R}$ is a smooth, compactly supported function such that $0 \notin \operatorname{supp} \psi$ and

$$
\mathcal{K}(t, x) \stackrel{\text { def }}{=} \int_{\mathbb{R}^{2}} e^{i x \cdot \xi} e^{ \pm i t|\xi|} \psi(\xi) d \xi
$$

then

$$
\|\mathcal{K}(t, \cdot)\|_{L^{\infty}} \lesssim \frac{1}{\sqrt{t}}
$$

for all $t>0$. This is done by some sort of integration by parts.

Next, let $\mathcal{C}$ be the support of the function $\varphi$ defined by (A.3), and consider a function $h$ such that $\operatorname{supp} h \subset \mathcal{C}$. On one hand,

$$
\left\|e^{ \pm i t|D|} h\right\|_{L^{\infty}} \lesssim \frac{1}{\sqrt{t}}\|h\|_{L^{1}}
$$

Indeed, let $\psi \in C_{0}^{\infty}\left(\mathbb{R}^{2}\right)$ equal to 1 on $\mathcal{C}$ and vanishing near the origin; then

$$
\begin{aligned}
\left\|e^{ \pm i t|D|} h\right\|_{L^{\infty}} & =\left\|e^{ \pm i t|D|} \psi(D) h\right\|_{L^{\infty}} \\
& \leq\left\|e^{ \pm i t|D|} \mathcal{F}^{-1} \psi\right\|_{L^{\infty}}\|h\|_{L^{1}},
\end{aligned}
$$


and (B.9) follows from (B.8). On the other hand,

$$
\left\|e^{ \pm i t|D|} h\right\|_{L^{2}}=\|h\|_{L^{2}}
$$

by Plancherel's formula. Interpolating between (B.9) and (B.10) gives

$$
\left\|e^{ \pm i t|D|} h\right\|_{L^{p}} \lesssim t^{-\frac{1}{2}+\frac{1}{p}}\|h\|_{L^{\bar{p}}},
$$

for all $p \in[2,+\infty]$.

Now comes the $T T^{\star}$ argument. Let $\bar{p}=p /(p-1), \bar{r}=r /(r-1)$, and

$$
\mathcal{B} \stackrel{\text { def }}{=}\left\{\Psi \in C_{0}^{\infty}\left(\mathbb{R}^{+} ; L_{x}^{\bar{p}}\right) ;\|\Psi\|_{L_{t}^{\bar{r}}\left(L_{x}^{\bar{p}}\right)} \leq 1\right\} .
$$

Then

$$
\begin{aligned}
\left\|e^{ \pm i t|D|} h\right\|_{L_{t}^{r}\left(L_{x}^{p}\right)} & =\sup _{\Psi \in \mathcal{B}} \int_{0}^{\infty}\left(e^{ \pm i t|D|} h, \Psi(t)\right)_{L^{2}} d t \\
& =\sup _{\Psi \in \mathcal{B}} \int_{0}^{\infty}\left(h, e^{\mp i t|D|} \psi(D) \Psi(t)\right)_{L^{2}} d t \\
& \leq\|h\|_{L^{2}} \alpha,
\end{aligned}
$$

with the same $\psi$ as above and

$$
\alpha \stackrel{\text { def }}{=} \sup _{\Psi \in \mathcal{B}}\left\|\int_{0}^{\infty} e^{\mp i t|D|} \psi(D) \Psi(t) d t\right\|_{L^{2}} .
$$

We have

$$
\begin{aligned}
\alpha^{2} & =\sup _{\Psi \in \mathcal{B}}\left(\int_{0}^{\infty} e^{\mp i t|D|} \psi(D) \Psi(t) d t, \int_{0}^{\infty} e^{\mp i \frac{s}{\epsilon}|D|} \psi(D) \Psi\left(t^{\prime}\right) d t^{\prime}\right)_{L^{2}} \\
& =\sup _{\Psi \in \mathcal{B}} \int_{0}^{\infty} \int_{0}^{\infty}\left(\psi(D) \Psi(t), e^{ \pm i \frac{t-t^{\prime}}{\epsilon}|D|} \psi(D) \Psi\left(t^{\prime}\right)\right)_{L^{2}} d t^{\prime} d t \\
& \lesssim \sup _{\Psi \in \mathcal{B}} \int_{0}^{\infty} \int_{0}^{\infty}\|\psi(D) \Psi(t)\|_{L^{\bar{p}}} \frac{1}{\left|t-t^{\prime}\right|^{\frac{1}{2}-\frac{1}{p}}}\left\|\psi(D) \Psi\left(t^{\prime}\right)\right\|_{L^{\bar{p}}} d t^{\prime} d t,
\end{aligned}
$$

applying (B.11). Hence, by Hölder inequality,

$$
\alpha^{2} \lesssim\left\|\int_{0}^{\infty} \frac{1}{\left|t-t^{\prime}\right|^{\frac{1}{2}-\frac{1}{p}}}\right\| \psi(D) \Psi\left(t^{\prime}\right)\left\|_{L^{\bar{p}}} d t^{\prime}\right\|_{L_{t}^{r}} .
$$

from which, using Hardy-Littlewood-Sobolev inequality [17], we deduce

$$
\left\|e^{ \pm i t|D|} h\right\|_{L_{t}^{r}\left(L_{x}^{p}\right)} \lesssim\|h\|_{L^{2}} .
$$

Finally, for all $m \in \mathbb{Z}$, set

$$
\begin{aligned}
\dot{\Delta}_{m} & =\varphi\left(2^{-m} D\right), \\
f_{m}(t, x) & =\left(\dot{\Delta}_{m} f\right)\left(2^{-m} t, 2^{-m} x\right), \\
f_{0, m} & =\left(\dot{\Delta}_{m} f_{0}\right)\left(2^{-m} \cdot\right), \\
g_{m}(t, x) & =2^{-m}\left(\dot{\Delta}_{m} g\right)\left(2^{-m} t, 2^{-m} x\right) .
\end{aligned}
$$


As $f_{m}$ is supported in $\mathcal{C}$ and satisfies

$$
\left\{\begin{array}{l}
\partial_{t} f_{m} \pm i|D| f_{m}=g_{m} \\
\left.f_{m}\right|_{t=0}=f_{0, m}
\end{array}\right.
$$

so that, by Duhamel's formula,

$$
f_{m}(t)=e^{\mp i t|D|} f_{0, m}+\int_{0}^{t} e^{\mp i\left(t-t^{\prime}\right)|D|} g_{m}\left(t^{\prime}\right) d t^{\prime}
$$

we get from (B.12)

$$
\left\|f_{m}\right\|_{L_{t}^{r}\left(L_{x}^{p}\right)} \lesssim\left\|f_{0, m}\right\|_{L^{2}}+\int_{0}^{t}\left\|g_{m}\left(t^{\prime}\right)\right\|_{L^{2}} d t^{\prime} .
$$

The changes of variables give

$$
\left\|\dot{\Delta}_{m} f\right\|_{L_{t}^{r}\left(L_{x}^{p}\right)} \lesssim 2^{m\left(\frac{3}{4}-\frac{3}{2 p}\right)}\left(\left\|\dot{\Delta}_{m} f_{0}\right\|_{L^{2}}+\int_{0}^{t}\left\|\dot{\Delta}_{m} g\left(t^{\prime}\right)\right\|_{L^{2}} d t^{\prime}\right)
$$

and therefore, if $q \leq 4$,

$$
\|f\|_{L_{t}^{r}\left(\dot{B}_{p, q}^{s}\right)} \lesssim\left\|f_{0}\right\|_{\dot{B}_{2, q}^{s+\frac{3}{4}-\frac{3}{2 p}}}+\int_{0}^{t}\left\|g\left(t^{\prime}\right)\right\|_{\dot{B}_{2, q}^{s+\frac{3}{4}-\frac{3}{2 p}}} d t^{\prime} .
$$

If $f_{0} \in L^{2}$ and $g \in L_{t}^{1}\left(L^{2}\right)$, then $f(t) \in L^{2}$ for all $t \geq 0$, so $\sum_{m=-\infty}^{-1} \dot{\Delta}_{m} f(t)$ converges to $\chi(D) f(t)$ in $L^{\infty}$. Hence,

$$
\|f(t)\|_{L^{p}} \lesssim\|f(t)\|_{\dot{B}_{p, 1}^{0}}
$$

and

$$
\|f(t)\|_{C^{s+1}} \lesssim\|f(t)\|_{\dot{B}_{\infty, q}^{0}}+\|f(t)\|_{\dot{B}_{\infty, q}^{s+1}}
$$

for any $q \in[1,+\infty]$; thus (B.2) and (B.3) follow from (B.13).

\section{Evolution of the curl}

The curl of $\tilde{v}_{\epsilon}=U_{\epsilon}^{\prime}+\sigma^{\prime}$, denoted by $\Omega_{\epsilon}$, satisfies

$$
\left\{\begin{array}{l}
\partial_{t} \Omega_{\epsilon}+\left(U_{\epsilon}^{\prime}+\sigma^{\prime}\right) \cdot \nabla \Omega_{\epsilon}+\Omega_{\epsilon} \operatorname{div} U_{\epsilon}^{\prime}=0 \\
\left.\Omega_{\epsilon}\right|_{t=0}=\Omega_{0, \epsilon}=\operatorname{rot} \tilde{v}_{0, \epsilon}
\end{array}\right.
$$

so (3.6) follows from the classical definition of Hölder spaces [2, p. 339]; but note that the techniques of dyadic decomposition [3, Lemma 4.1.1], give in addition estimates like (3.6) for plenty of $s$ outside $] 0,1[$.

Since

$$
\Omega_{\epsilon}(t, x)=\Omega_{0, \epsilon}\left(\psi_{\epsilon}^{-1}(t, x)\right)-\int_{0}^{t}\left(\Omega_{\epsilon} \operatorname{div} U_{\epsilon}^{\prime}\right)\left(t^{\prime}, \psi_{\epsilon}\left(t^{\prime}, \psi_{\epsilon}^{-1}(t, x)\right)\right) d t^{\prime}
$$


if $\psi_{\epsilon}$ is the flow of $\tilde{v}_{\epsilon}$, that is, the solution of

$$
\left\{\begin{array}{l}
\partial_{t} \psi_{\epsilon}(t, x)=\tilde{v}_{\epsilon}\left(t, \psi_{\epsilon}(t, x)\right) \\
\psi_{\epsilon}(0, x)=x
\end{array}\right.
$$

we also have

$$
\begin{aligned}
\left\|\Omega_{\epsilon}(t)\right\|_{L^{p}} \leq & \left\|\Omega_{0, \epsilon} \circ \psi_{\epsilon}^{-1}(t, \cdot)\right\|_{L^{p}} \\
& +\int_{0}^{t}\left\|\operatorname{div} U_{\epsilon}^{\prime}\left(t^{\prime}\right)\right\|_{L^{\infty}}\left\|\Omega_{\epsilon}\left(t^{\prime}\right) \circ \psi_{\epsilon}\left(t^{\prime}, \psi_{\epsilon}^{-1}(t, \cdot)\right)\right\|_{L^{p}} d t^{\prime} \\
\leq & \left\|\Omega_{0, \epsilon}\right\|_{L^{p}}\left\|\operatorname{det} \nabla \psi_{\epsilon}(t, \cdot)\right\|_{L^{\infty}}^{\frac{1}{p}} \\
& +\int_{0}^{t}\left\|\operatorname{div} U_{\epsilon}^{\prime}\left(t^{\prime}\right)\right\|_{L^{\infty}}\left\|\Omega_{\epsilon}\left(t^{\prime}\right)\right\|_{L^{p}}\left\|\operatorname{det} \nabla \psi_{\epsilon}^{-1}\left(t^{\prime}, \psi_{\epsilon}^{-1}(t, \cdot)\right)\right\|_{L^{\infty}}^{\frac{1}{p}} d t^{\prime} \\
\leq & \left\|\Omega_{0, \epsilon}\right\|_{L^{p}} e^{\frac{1}{p} \int_{0}^{t}\left\|\operatorname{div} \tilde{v}_{\epsilon}\left(t^{\prime}\right)\right\|_{L^{\infty}} d t^{\prime}} \\
& +\int_{0}^{t}\left\|\operatorname{div} U_{\epsilon}^{\prime}\left(t^{\prime}\right)\right\|_{L^{\infty}}\left\|\Omega_{\epsilon}\left(t^{\prime}\right)\right\|_{L^{p}} e^{\frac{1}{p} \int_{t^{\prime}}^{t}\left\|\operatorname{div} \tilde{v}_{\epsilon}\left(t^{\prime \prime}\right)\right\|_{L^{\infty}} d t^{\prime \prime}} d t^{\prime},
\end{aligned}
$$

using the basic result on the evolution of a flow's Jacobian [16, 18]. Hence we get (3.5) by Gronwall's lemma, as $\operatorname{div} \tilde{v}_{\epsilon}=\operatorname{div} U_{\epsilon}^{\prime}$.

\section{Acknowledgments}

A. Dutrifoy's research was supported through a European Community Marie Curie Fellowship. http://www.cordis.lu.improving Disclaimer: The author is solely responsible for information communicated and the European Commission is not responsible for any view or results expressed.

\section{References}

[1] K. Asano. On the incompressible limit of the compressible Euler equation. Japan J. Appl. Math., 4(3):455-488, 1987.

[2] H. Bahouri and B. Dehman. Remarques sur l'apparition de singularités dans les écoulements eulériens incompressibles à donnée initiale höldérienne. J. Math. Pures Appl., 73:335-346, 1994.

[3] J.-Y. Chemin. Perfect incompressible fluids, volume 14 of Oxford Lecture Series in Mathematics and its Applications. The Clarendon Press Oxford University Press, New York, 1998. Translated from the 1995 French original by Isabelle Gallagher and Dragos Iftimie.

[4] J.-Y. Chemin, B. Desjardins, I. Gallagher, and E. Grenier. Anisotropy and dispersion in rotating fluids. Preprint of Université d'Orsay, 1999. Available as http://www.math.u-psud.fr/ biblio/pub/1999/fic/ ppo_1999_69.ps. 
[5] J.-Y. Chemin, B. Desjardins, I. Gallagher, and E. Grenier. Fluids with anisotropic viscosity. M2AN Math. Model. Numer. Anal., 34(2):315335, 2000. Special issue for R. Temam's 60th birthday.

[6] R. Danchin. Zero Mach number limit in critical spaces for compressible Navier-Stokes equations. Ann. Sci. École Norm. Sup. (4), 35(1):27-75, 2002.

[7] B. Desjardins and E. Grenier. Low Mach number limit of viscous compressible flows in the whole space. R. Soc. Lond. Proc. Ser. A Math. Phys. Eng. Sci., 455(1986):2271-2279, 1999.

[8] B. Desjardins, E. Grenier, P.-L. Lions, and N. Masmoudi. Incompressible limit for solutions of the isentropic Navier-Stokes equations with Dirichlet boundary conditions. J. Math. Pures Appl. (9), 78(5):461471, 1999.

[9] A. Dutrifoy. Examples of dispersive effects in non-viscous rotating fluids. Submitted, July 2003.

[10] A. Dutrifoy. On 3-D vortex patches in bounded domains. Comm. Partial Differential Equations, 28(7\&8):1237-1263, 2003.

[11] A. Dutrifoy. Precise regularity results for the Euler equations. J. Math. Anal. Appl., 282(1):177-200, 2003.

[12] A. Dutrifoy. Slow convergence to vortex patches in quasigeostrophic balance. Submitted, March 2003.

[13] A. Dutrifoy and T. Hmidi. The incompressible limit of solutions of the two-dimensional compressible Euler system with degenerating initial data. C. R. Math. Acad. Sci. Paris, 336(6):471-474, 2003.

[14] P. Gamblin and X. Saint Raymond. On three-dimensional vortex patches. Bull. Soc. Math. France, 123(3):375-424, 1995.

[15] J. Ginibre and G. Velo. Generalized Strichartz inequalities for the wave equation. J. Funct. Anal., 133(1):50-68, 1995.

[16] P. Hartman. Ordinary Differential Equations. John Wiley \& Sons, 1964.

[17] L. Hörmander. The Analysis of Linear Partial Differential Operators, volume I. Springer-Verlag, 1983.

[18] L. Hörmander. Lectures on Nonlinear Hyperbolic Differential Equations, volume 26 of Mathématiques et Applications. Springer-Verlag, 1997. 
[19] S. Klainerman and A. Majda. Singular limits of quasilinear hyperbolic systems with large parameters and the incompressible limit of compressible fluids. Comm. Pure Appl. Math., 34(4):481-524, 1981.

[20] S. Klainerman and A. Majda. Compressible and incompressible fluids. Comm. Pure Appl. Math., 35(5):629-651, 1982.

[21] C.-K. Lin. On the incompressible limit of the slightly compressible viscous fluid flows. In Nonlinear waves (Sapporo, 1995), volume 10 of GAKUTO Internat. Ser. Math. Sci. Appl., pages 277-282. Gakkōtosho, Tokyo, 1997.

[22] P.-L. Lions and N. Masmoudi. Incompressible limit for a viscous compressible fluid. J. Math. Pures Appl. (9), 77(6):585-627, 1998.

[23] A. Majda. Compressible fluid flow and systems of conservation laws in several space variables, volume 53 of Applied Mathematical Sciences. Springer-Verlag, New York, 1984.

[24] G. Métivier and S. Schochet. The incompressible limit of the nonisentropic Euler equations. Arch. Ration. Mech. Anal., 158(1):61-90, 2001.

[25] H. Triebel. Theory of function spaces, volume 78 of Monographs in Mathematics. Birkhäuser Verlag, Basel, 1983.

[26] S. Ukai. The incompressible limit and the initial layer of the compressible Euler equation. J. Math. Kyoto Univ., 26(2):323-331, 1986. 